\title{
American Indian Pottery from Historic Period Sites in North Louisiana
}

Hiram F. Gregory

Unknown

George Avery

Follow this and additional works at: https://scholarworks.sfasu.edu/ita

Part of the American Material Culture Commons, Archaeological Anthropology Commons, Environmental Studies Commons, Other American Studies Commons, Other Arts and Humanities Commons, Other History of Art, Architecture, and Archaeology Commons, and the United States History Commons

Tell us how this article helped you.

This Article is brought to you for free and open access by the Center for Regional Heritage Research at SFA ScholarWorks. It has been accepted for inclusion in Index of Texas Archaeology: Open Access Gray Literature from the Lone Star State by an authorized editor of SFA ScholarWorks. For more information, please contact cdsscholarworks@sfasu.edu. 


\section{American Indian Pottery from Historic Period Sites in North Louisiana}

Creative Commons License

(c) (i) (8)

This work is licensed under a Creative Commons Attribution-NonCommercial 4.0 International License 


\title{
AMERICAN INDIAN POTTERY FROM HISTORIC PERIOD SITES IN NORTH LOUISIANA
}

\author{
H. F. "Pete" Gregory and George Avery
}

The following is a revised version of a presentation given at the annual meeting of the Louisiana Archaeological Society (LAS) held in Monroe, Louisiana, on March 4, 2006 (Gregory and Avery 2006). The goal of the LAS presentation was to simply show photographs and illustrations of American Indian pottery that dates to the historic period in North Louisiana. Most of the photographs and illustrations are from a manuscript written by H.F. "Pete" Gregory entitled Los Adaes (I6NA16) American Indian Ceramics (2005). This manuscript was compiled by George Avery as part of the Los Adaes Station Archaeology Program, funded entirely by the Louisiana Division of Archaeology. The LAS presentation was given again at the December 2, 2006 meeting of the East Texas Caddo Research Group (ETCRG) at Stephen F. Austin State University, Nacogdoches, Texas. Revisions to the LAS presentation for the current publication were made as a result of comments from the ETCRG meeting.

It should be noted that we are following R. K. Harris' definition for "Emory Punctated" (Story et al. 1967:136-139). Emory Punctated as defined by Harris at the Gilbert Site (4IRA13) refers to constricted neck vessels with two or more rows of punctates around the neck, and parallel curvilinear lines below the rows of punctates. We describe the sherds with one or more rows of punctations around the neck of a constricted neck vessel that lack incised lines below the row(s) of punctates as simply "Constricted Neck Punctated". As defined by Harris, certain elements of Emory Punctated are similar to those found on Foster Trailed Incised and Winterville Incised, var. Tunica vessels (see Girard 2006:28-29). Also, the constricted neck punctated forms are similar to the Tunica Mode described by Jeffrey Brain which occurs on Winterville Incised, var. Tunica (Brain 1979:234-235). Brain (1979:229) also illustrates an otherwise plain vessel with a Tunica mode from the Trudeau site (16WF25). The current interpretation of Emory Punctated for Texas materials (e.g. Perttula 2005:186) differs from the interpretation of Emory Punctated for Los Adaes materials (Gregory 2005). The Emory Punctated at Los Adaes was graciously referred to as "Louisiana Emory" at the ETCRG by a Texas colleague. Clearly, a more thorough investigation and discussion of the "overlap" between Emory Punctated, Foster Trailed Incised, Winterville Incised, and the Tunica Mode is in order, and hopefully the following presentation will spark such investigation and discussion.

The following presentation starts with a distribution table for various historic period pottery types along with their geographic distribution, and the names of the American Indian groups that were in these areas during the historic period. The pottery is presented starting with examples of engraved surface treatment, followed by incised, punctated, punctated-incised, combed, plain, and plain with European influences. It should be noted that many of the "plain" examples exhibit a single incised line or raised area around the neck, which effectively separates the rim and body. Frank Schambach described the upper/lower delineation for decorated Caddo pottery as the "Rule of 'Two." 
The "Rule of Two" also seems to be present on wares that have no other surface design elements except for the upper/lower delineation in the form of an incised line or raised area. Such vessels are described here as "Rule of Two" Plain, which are present at Los Adaes, the Trudeau site (16WF25) (Brain 1979:229-231), and the Procello site (16DS212) (Espey, Huston \& Associates 1983: Figure 26a). Finally, plain wares with European influences are depicted, such as the pitcher, plate, and porringer forms, and the foot ring attribute.

We wish to thank the following for their help:

Jeff Girard, NW Louisiana Regional Archaeologist

Louisiana Division of Archaeology

Bobby Gonzalez, NAGPRA Coordinator

Caddo Tribe of Oklahoma

Earl Barbry, Jr., NAGPRA Coordinator

Tunica-Biloxi Tribe of Louisiana

Gary Hardamon, NSU Supervisor of Photography under contract with the

NW Louisiana Regional Archaeology Program

Shelly Witten and Josh Martin, NSU Anthropology Majors,

Artifact Illustrators employed by the

I.os Adaes Station Archaeology Program

\section{References Cited}

Brain, Jeffrey $P$.

1979 Tunica Treasure. The Peabody Museum, Harvard University, Cambridge, Massachusetts, and the Peabody Museum of Salem, Salem, Massachusetts.

Espey, Huston, \& Associates

1983 The Archaeological Investigation of the Louis Procello Sile, I6DS212,

DeSoto Parish, Louisiana. Document No. 82360, EH\&A Job No. 1731. Espey, Huston, \& Associates, Austin, Texas.

Girard, Jeffrey S.

2006 Regional Archaeology Program. Management Unit 1. Seventeenth Annual

Reporl 2006. Northwesterm State University of Louisiana, Natchitoches. 
Gregory, H.F.

2005 Los Adaes (16NA16) American Indian Ceramics. Manuscript compiled by George Avery, Los Adaes Station Archaeologist. Dept. of Social Sciences, Northwestern State University of Louisiana, Natchitoches.

Gregory, H.F. "Pete" and George Avery

2006 Using American Indian Pottery to Identify Historic Period Sites in Northwest Louisiana. Presented at the Annual Meeting of the Louisiana Archaeological Society, Monroe, Louisiana, March 4, 2006.

Perttula, Timothy K.

2005 Winston's Mounds and Shawnee Town: Mound Explorations by the Bureau of Ethnology in Texas, 1882-1884. Bulletin of the Texas Archeological Society 76:183-188.

Story, Dee Ann; Byron Barber, Estalee Barber, Evelyn Cobb, Hershel Cobb, Robert Coleman, Kathleen Gilmore, R.K. Harris, and Norma Hoffrichter

1967 Pottery Vessels. Bulletin of the Texas Archeological Society 37:112-188.

Webb, Clarence $\mathrm{H}$.

1945 A Second Historic Caddo site at Natchitoches, Louisiana. Bulletin of the Texas Archeological and Paleontological Society 16:52-83. 
Table 1. Distribution of Historic Period American Indian Pottery in North Louisiana

\begin{tabular}{|c|c|c|c|}
\hline & Natchitoches/NW & Ouachita Area & Lower Red River \\
\hline & Tribal Groups & Tribal Groups & Tribal Groups \\
\hline & \multirow[t]{2}{*}{$\begin{array}{l}\text { Natchitoches, Adaes } \\
\text { Yatasi, Natchez } \\
\text { Choctaw, Apalachee } \\
\text { Coushatta }\end{array}$} & \multirow[t]{2}{*}{$\begin{array}{c}\text { Ouachita } \\
\text { Koroa } \\
\text { Choctaw (later) } \\
\text { Pascagoula (later) }\end{array}$} & \multirow[t]{2}{*}{$\begin{array}{c}\text { Avoyel } \\
\text { Tunica-Biloxi }\end{array}$} \\
\hline Pottery Type/Description & & & \\
\hline Keno Trailed & $\bar{x}$ & $\bar{x}$ & $\mathrm{X}$ (rare) \\
\hline Natchitoches, Engraved & $x$ & $x$ & $x$ \\
\hline Patton Engraved & $X$ (rare) & 0 & 0 \\
\hline Emory Punctated & $\mathrm{x}$ & 0 & 0 \\
\hline Ebarb incised & $\mathrm{x}$ & 0 & 0 \\
\hline Fatherland Incised & $\mathrm{x}$ & $\mathbf{x}$ & $\mathrm{x}$ \\
\hline Chickachae Combed & $\mathrm{x}$ & 0 & 0 \\
\hline Coushatta "Pie Edge" rims & $x$ & 0 & 0 \\
\hline Zimmerman Plain & $\mathrm{x}$ & 0 & 0 \\
\hline Constricted Neck Punctated & $x$ & 0 & $x$ \\
\hline "Rule of Two" Plain & $\mathrm{x}$ & $\mathrm{x}$ & $x$ \\
\hline Plain with European Influences & $x$ & 0 & $\mathrm{x}$ \\
\hline
\end{tabular}


Journal of Northeast Texas Archaeology, No. 26 (2007)

0
0
0
0
0
0
0
0
0
0


क



\section{Natchitoches Engraved}

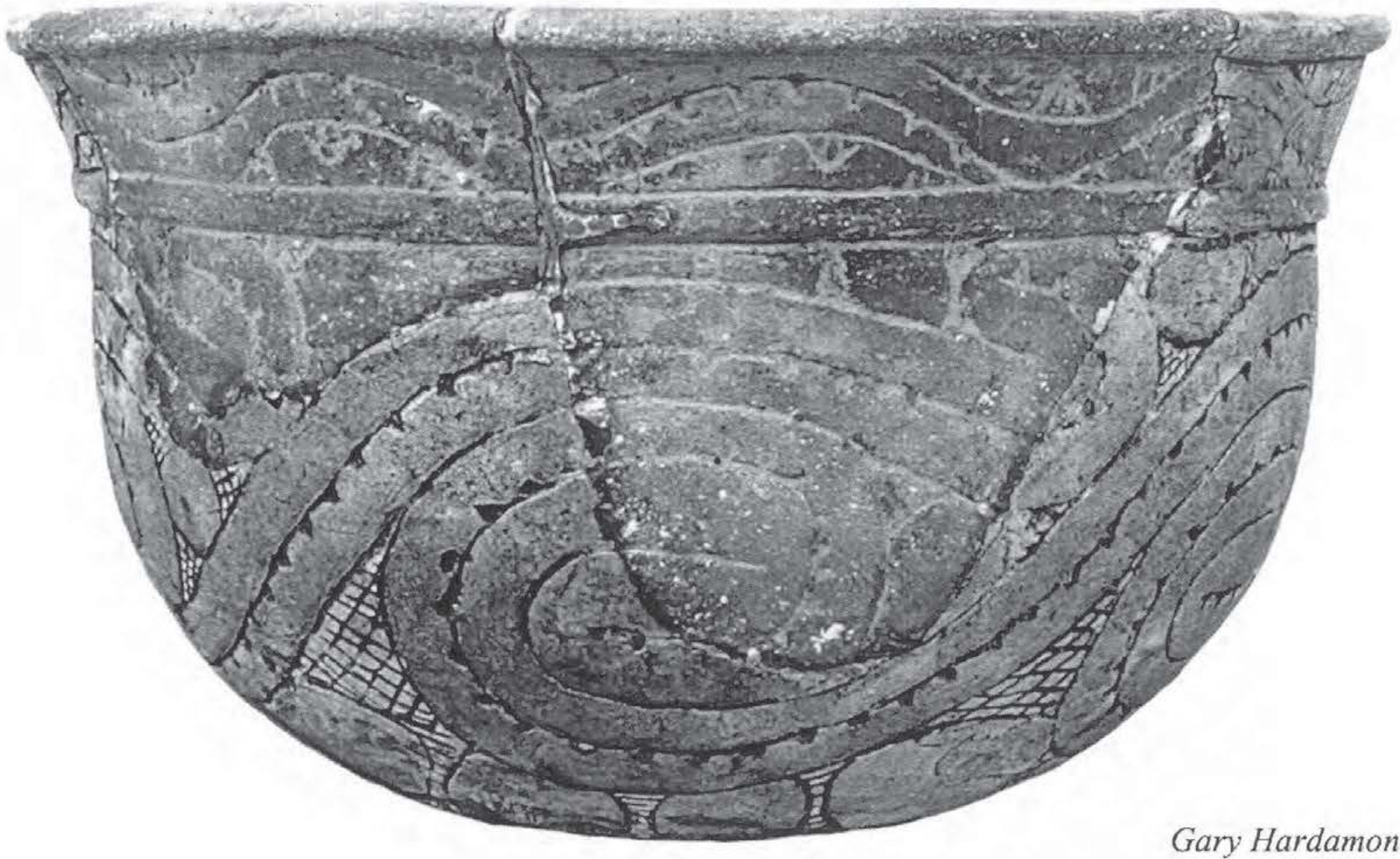


Journal of Northeast Texas Archaeology, No. 26 (2007)

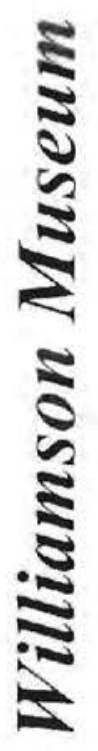
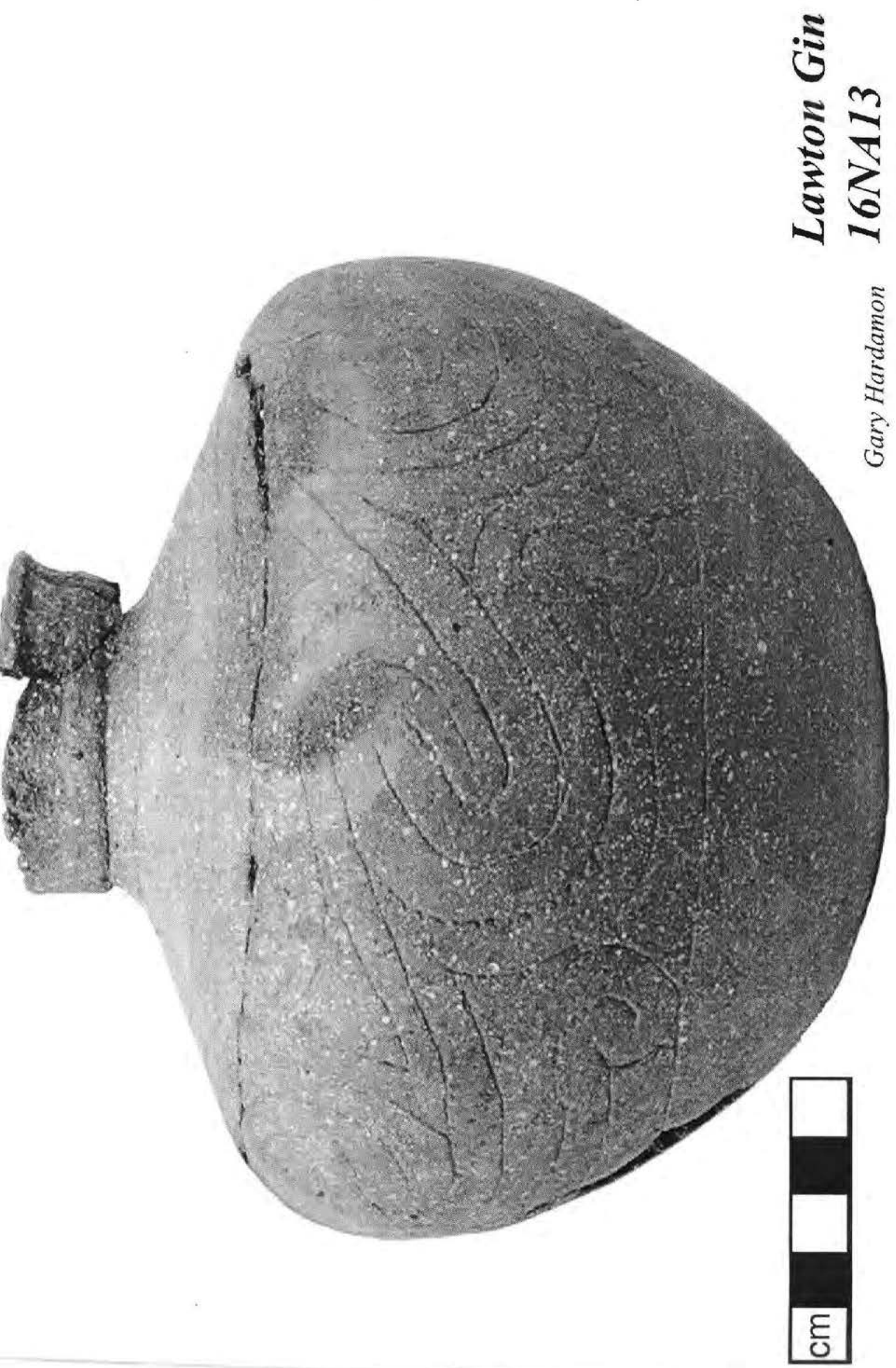


\section{Natchitoches Engraved}

(Gregory 2005 ms.)


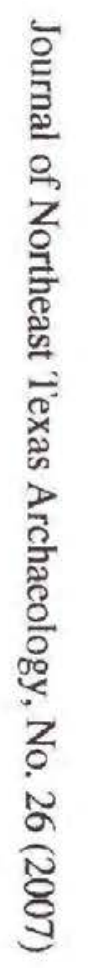



shell tempered

Los Adaes 16NA16 


\section{Natchitoches Engraved}

(Gregory 2005 ms.)
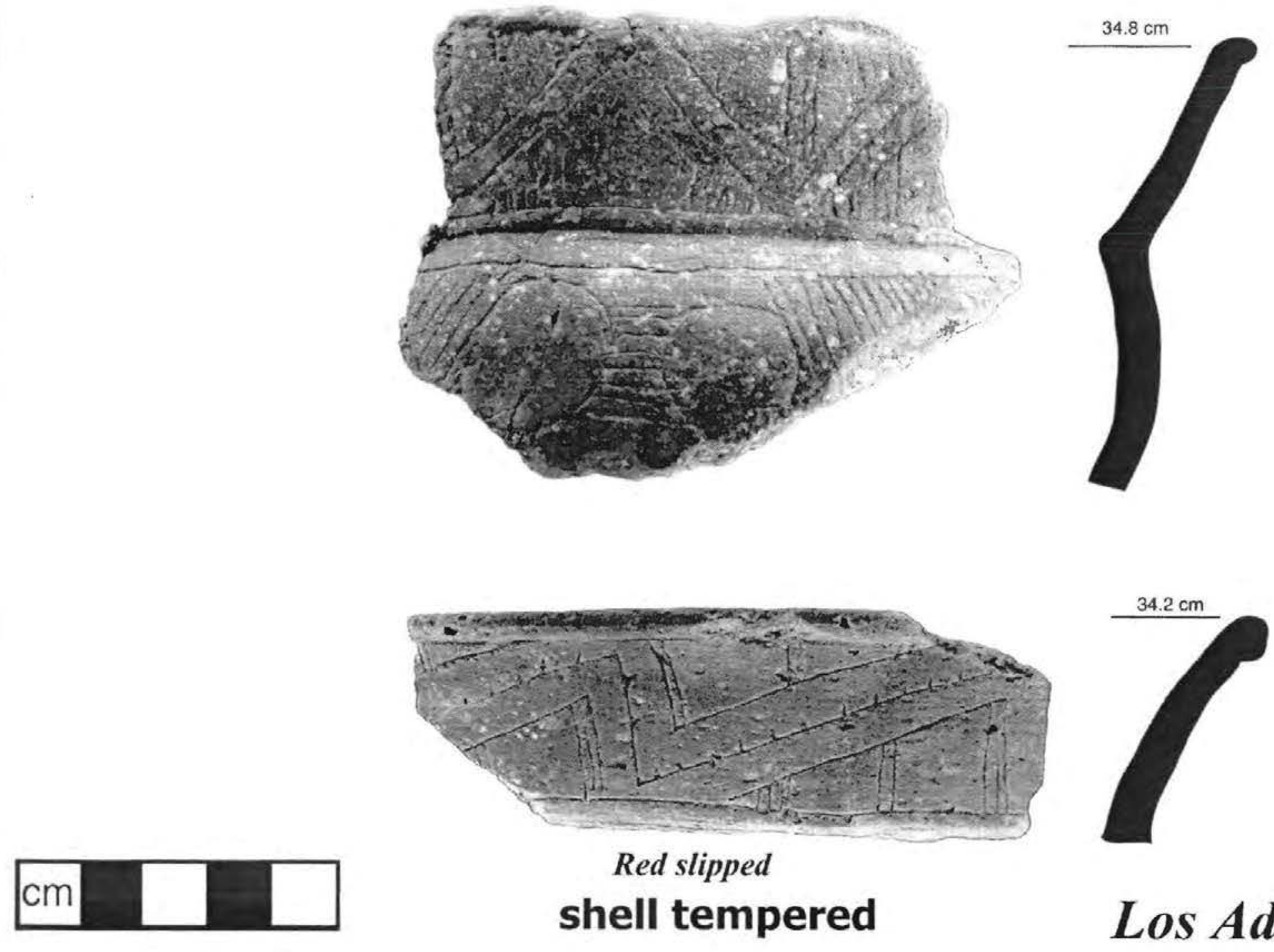






\section{Natchitoches Engraved}

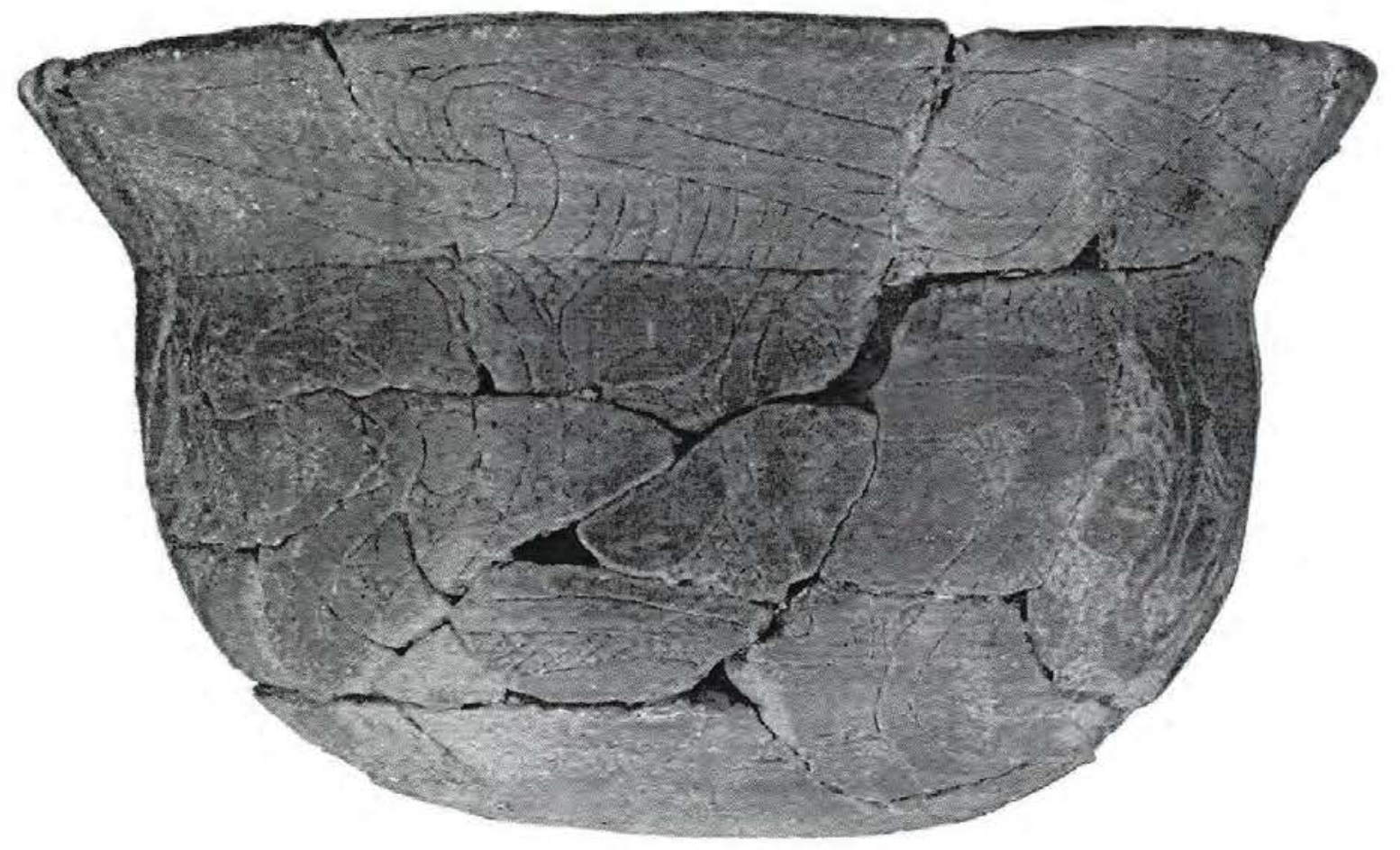

Red slipped 
Natchitoches Engraved

(Gregory 2005 ms.)
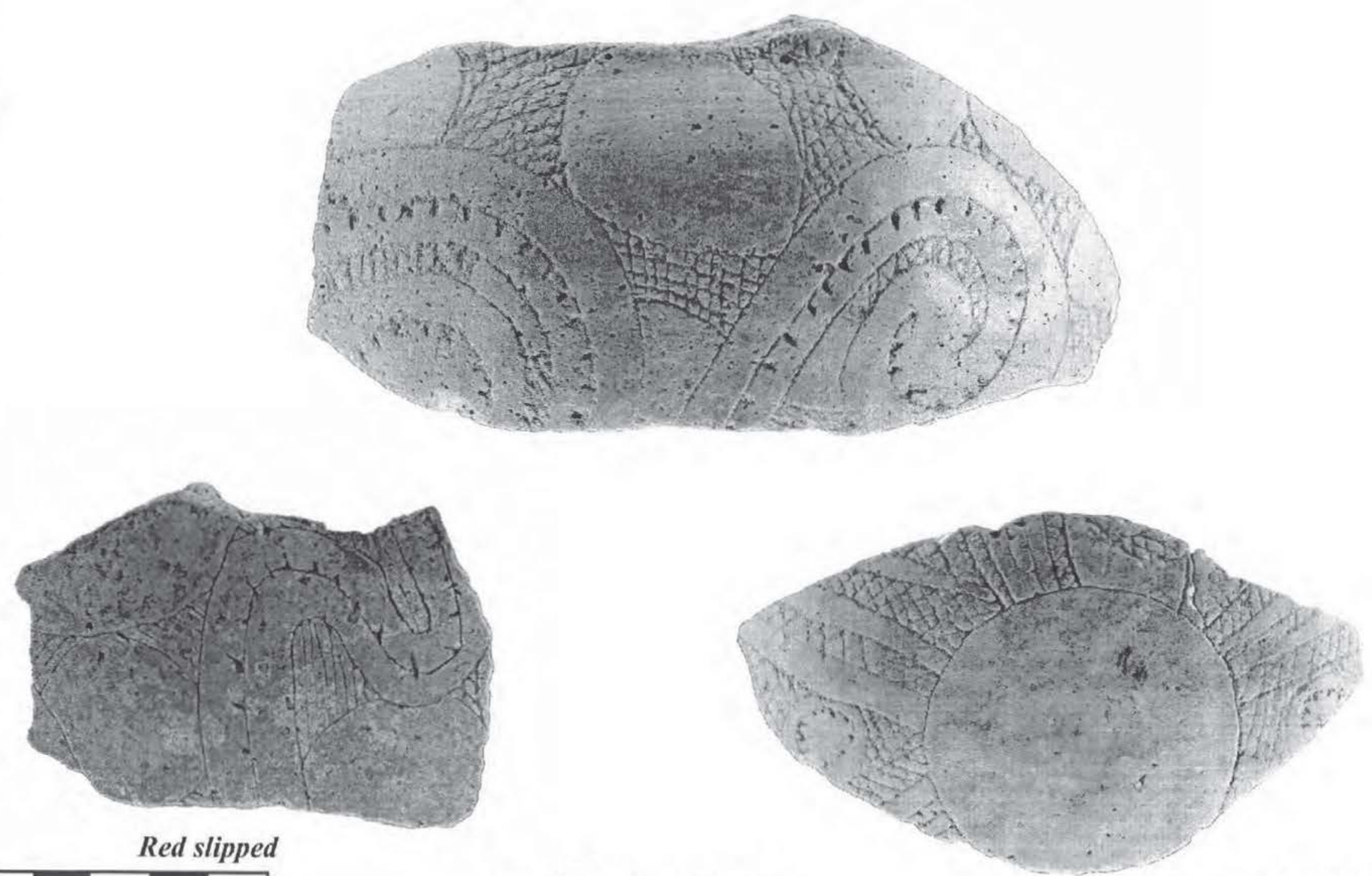

shell tempered

Los Adaes 16NA16 


\section{Patton Engraved}

(Gregory 2005 ms.)



\section{Ebarb Incised}

(Gregory 2005 ms.)

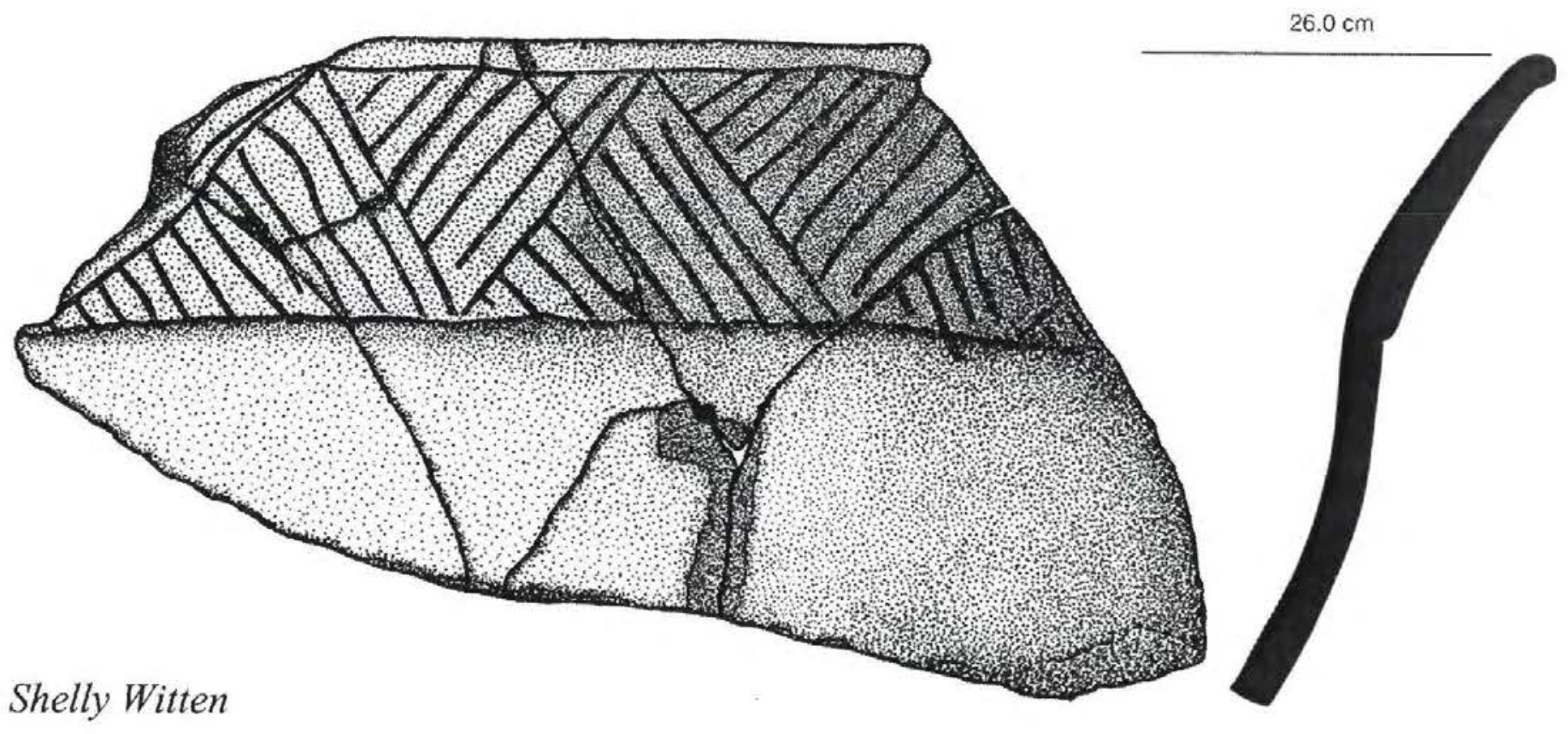

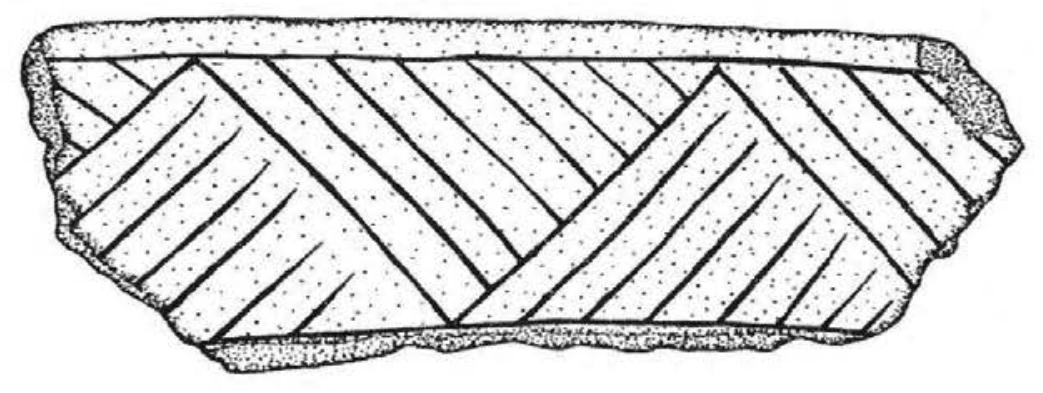

shell and bone tempered

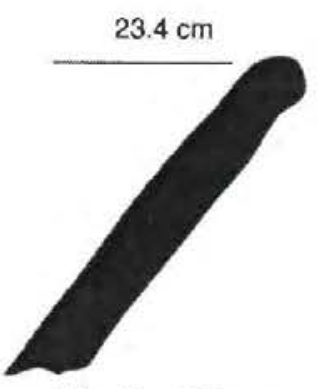

Shelly Witten

Los Adaes 16NA16 

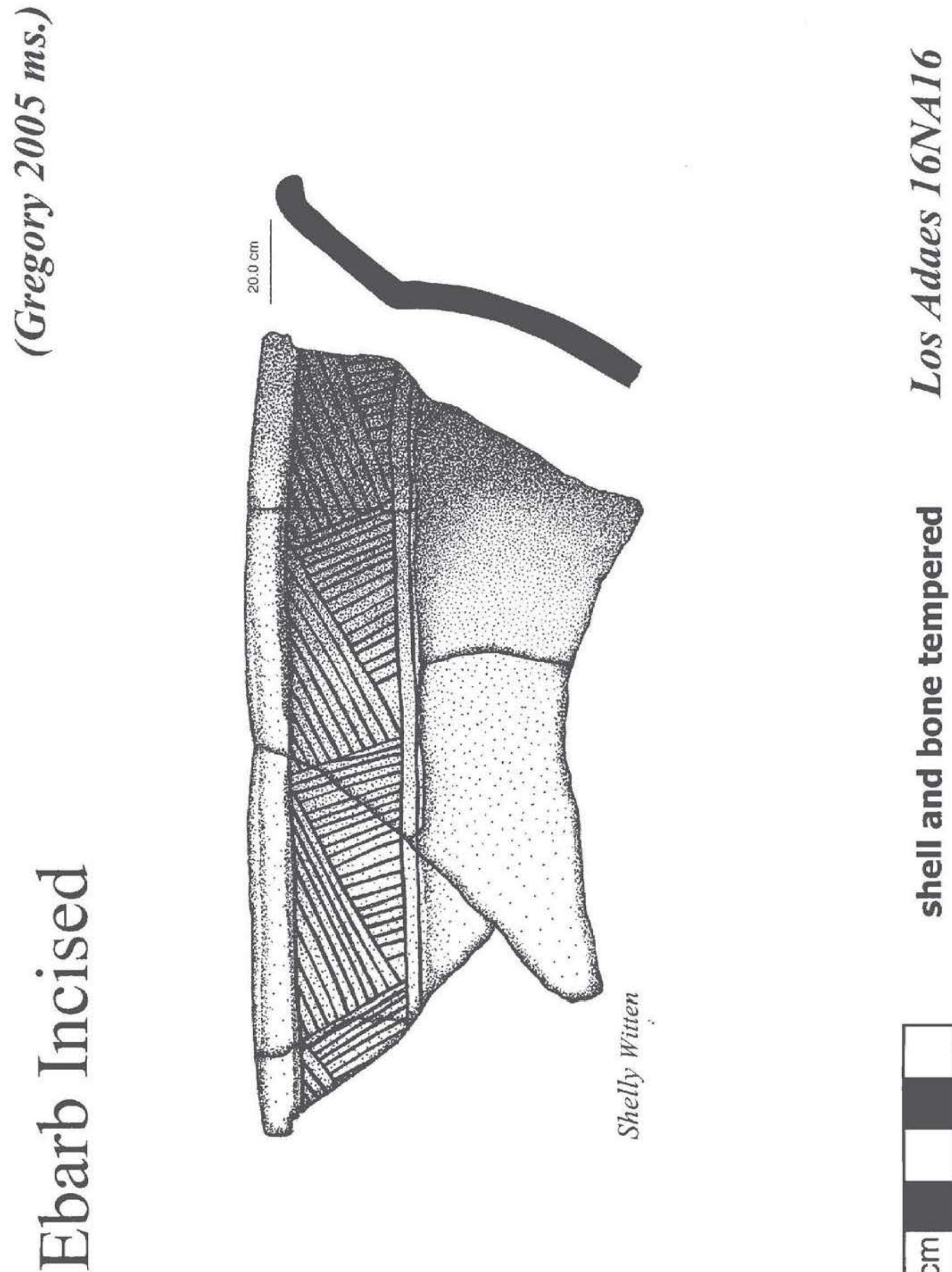


\section{Ebarb Incised}
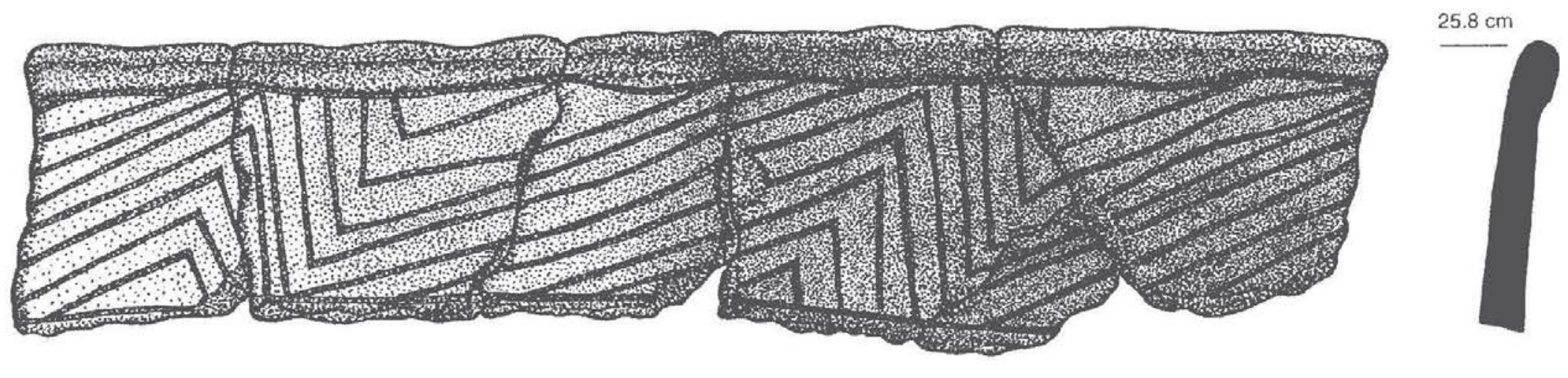

Shelly Witten
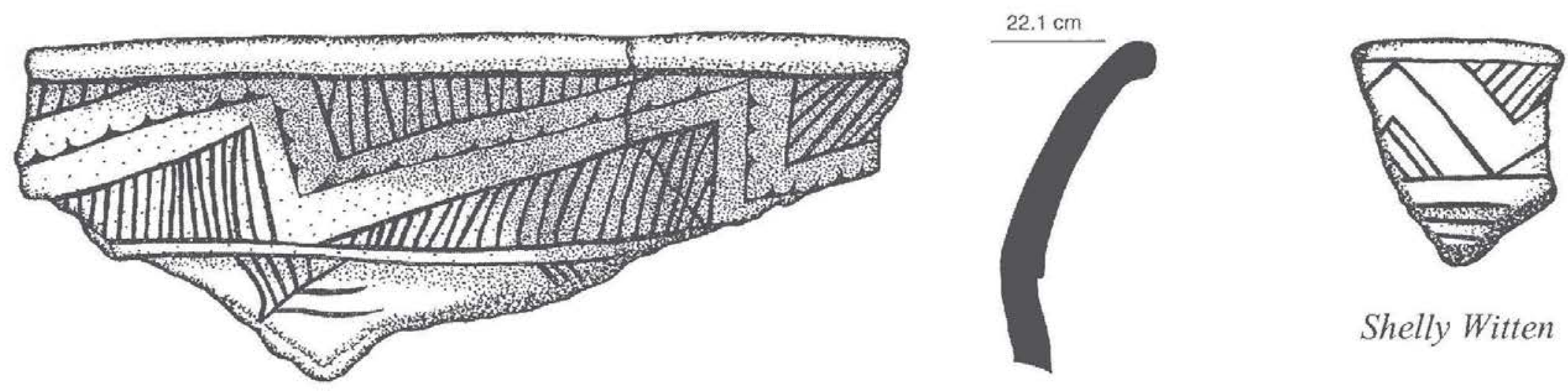

Shelly Witten

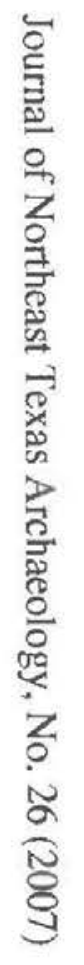

Shelly Witten

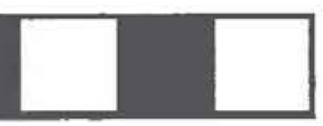

Los Adaes 16NA16 
2
0
0
0
0
0
0
0


2

$\frac{2}{8}$

8
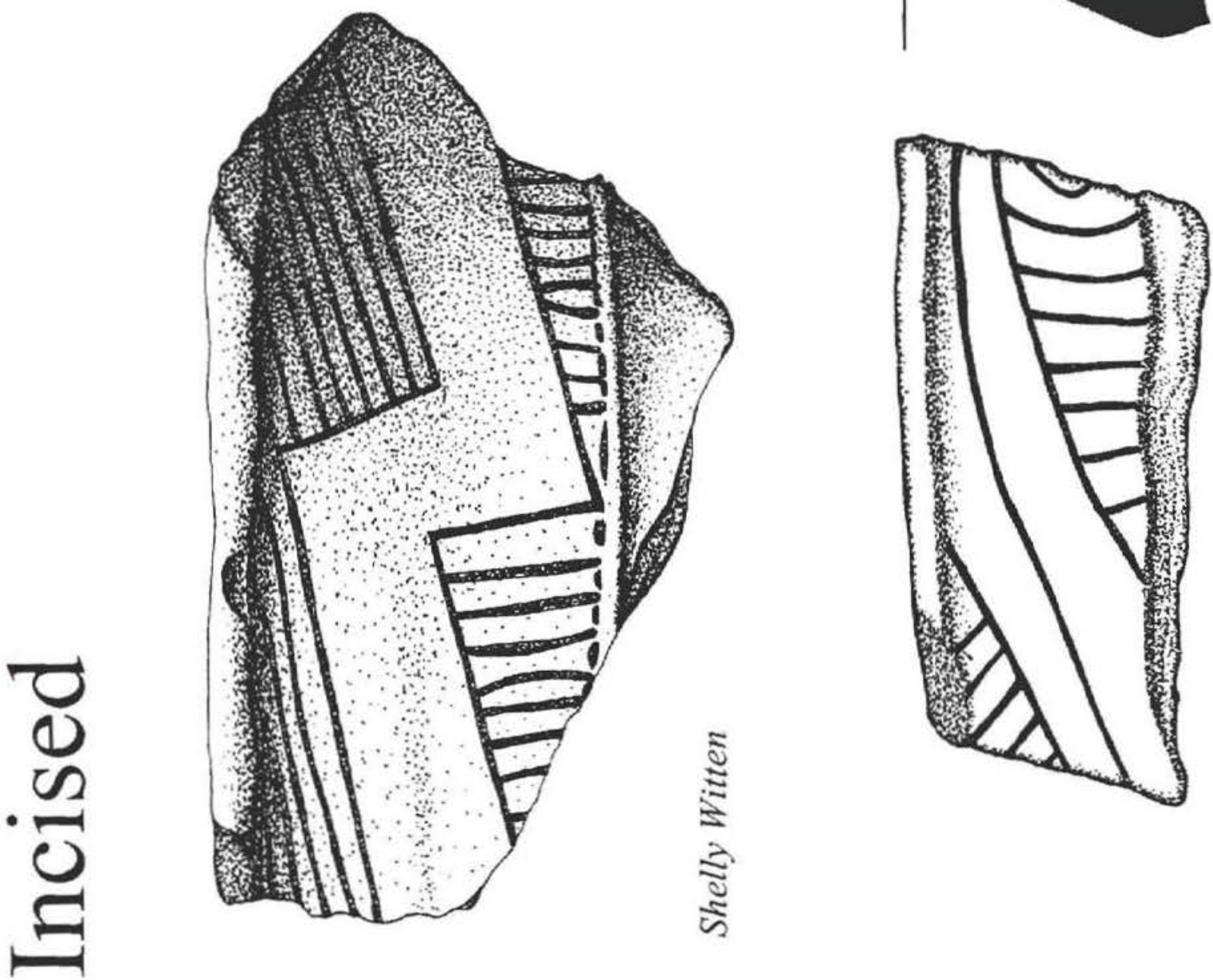

ڤั)
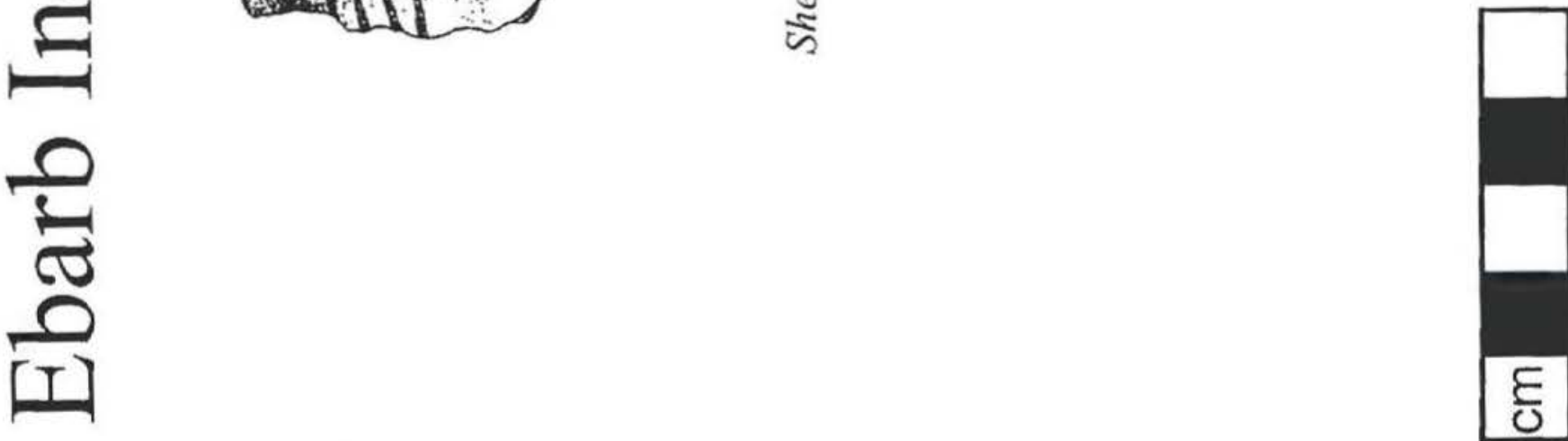


\section{Constricted Neck Punctated}

(Gregory 2005 ms.)

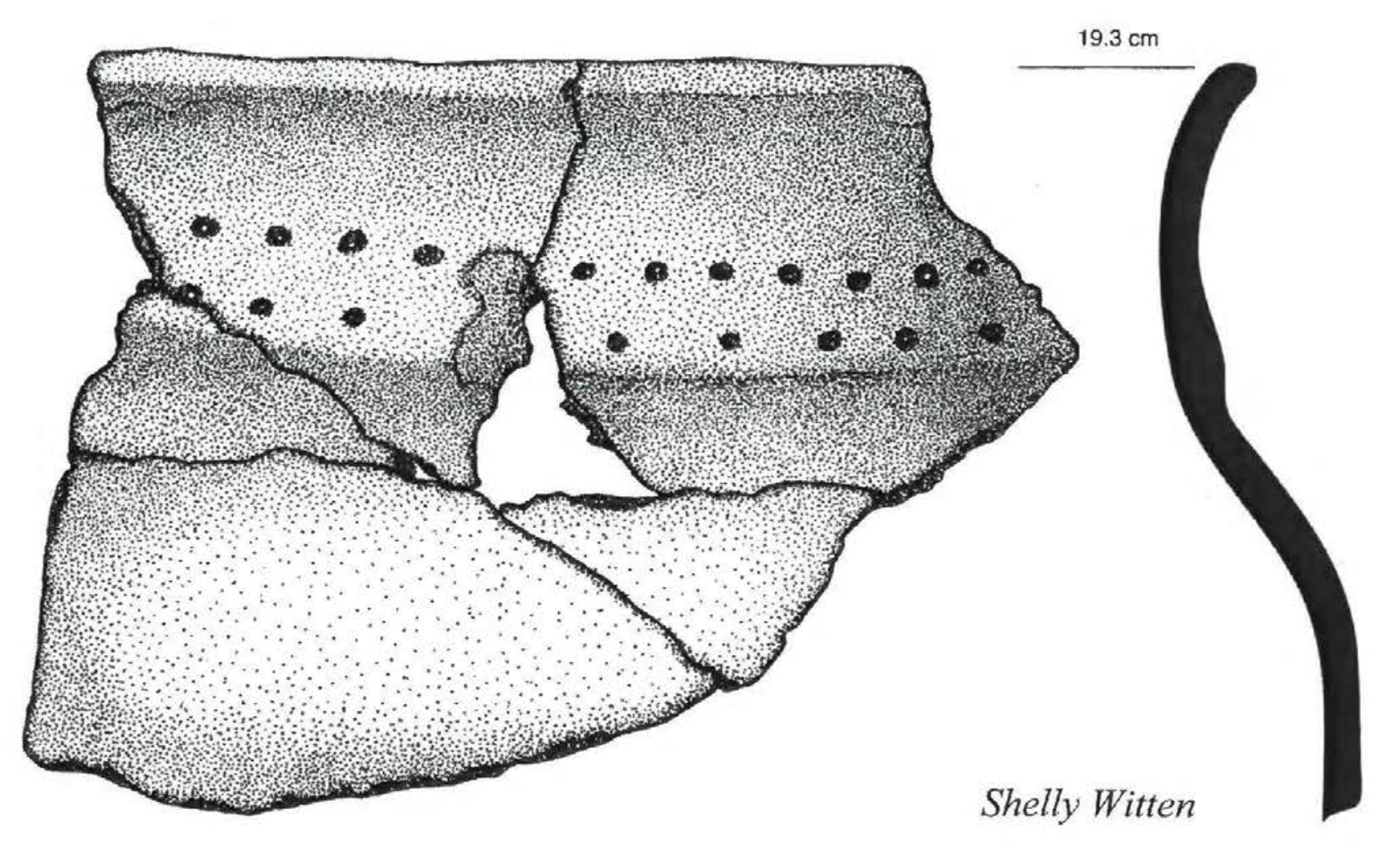




\section{Constricted Neck Punctated}

(Gregory 2005 ms.)
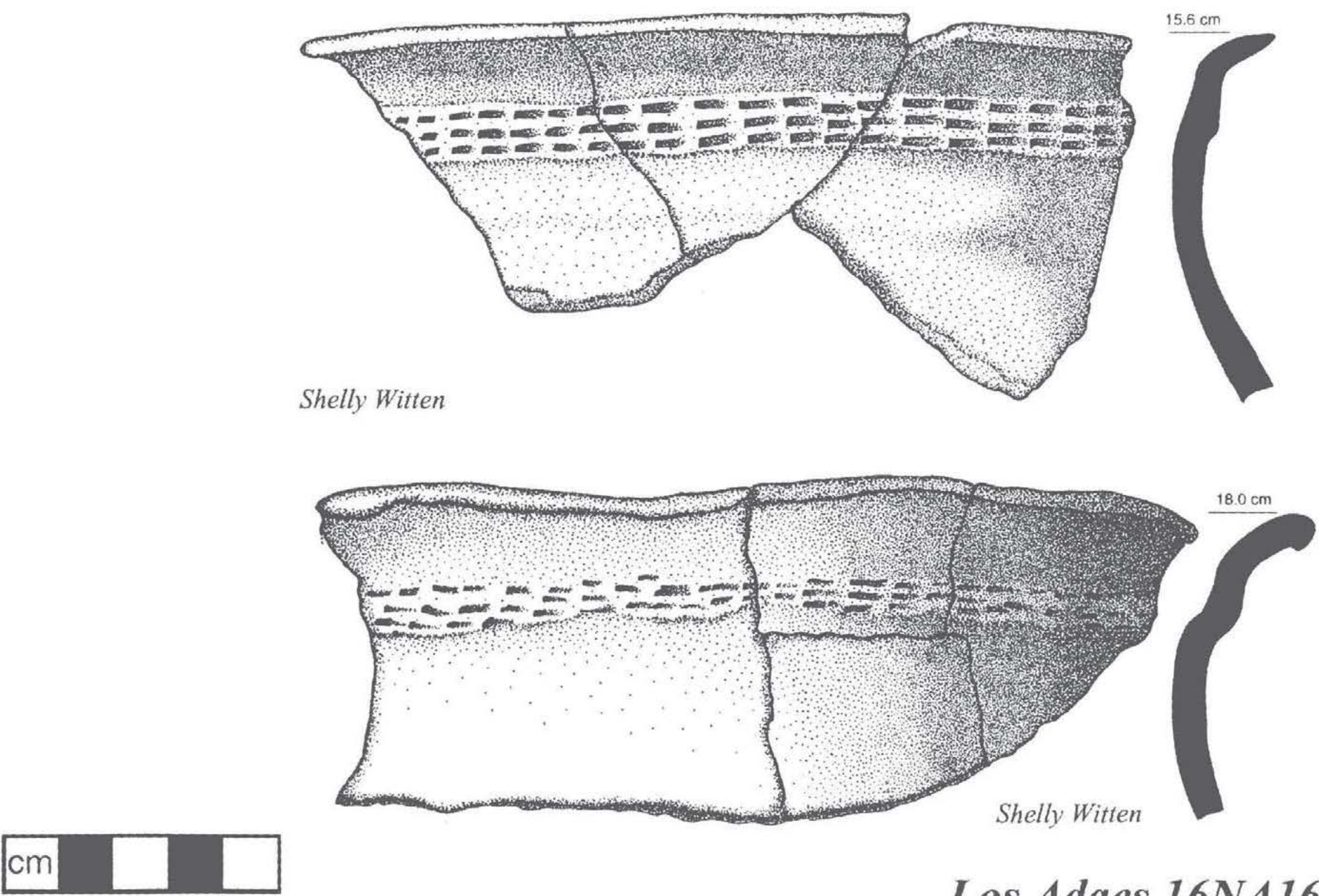


\section{Constricted Neck Punctated \\ (Gregory 2005 ms.)}

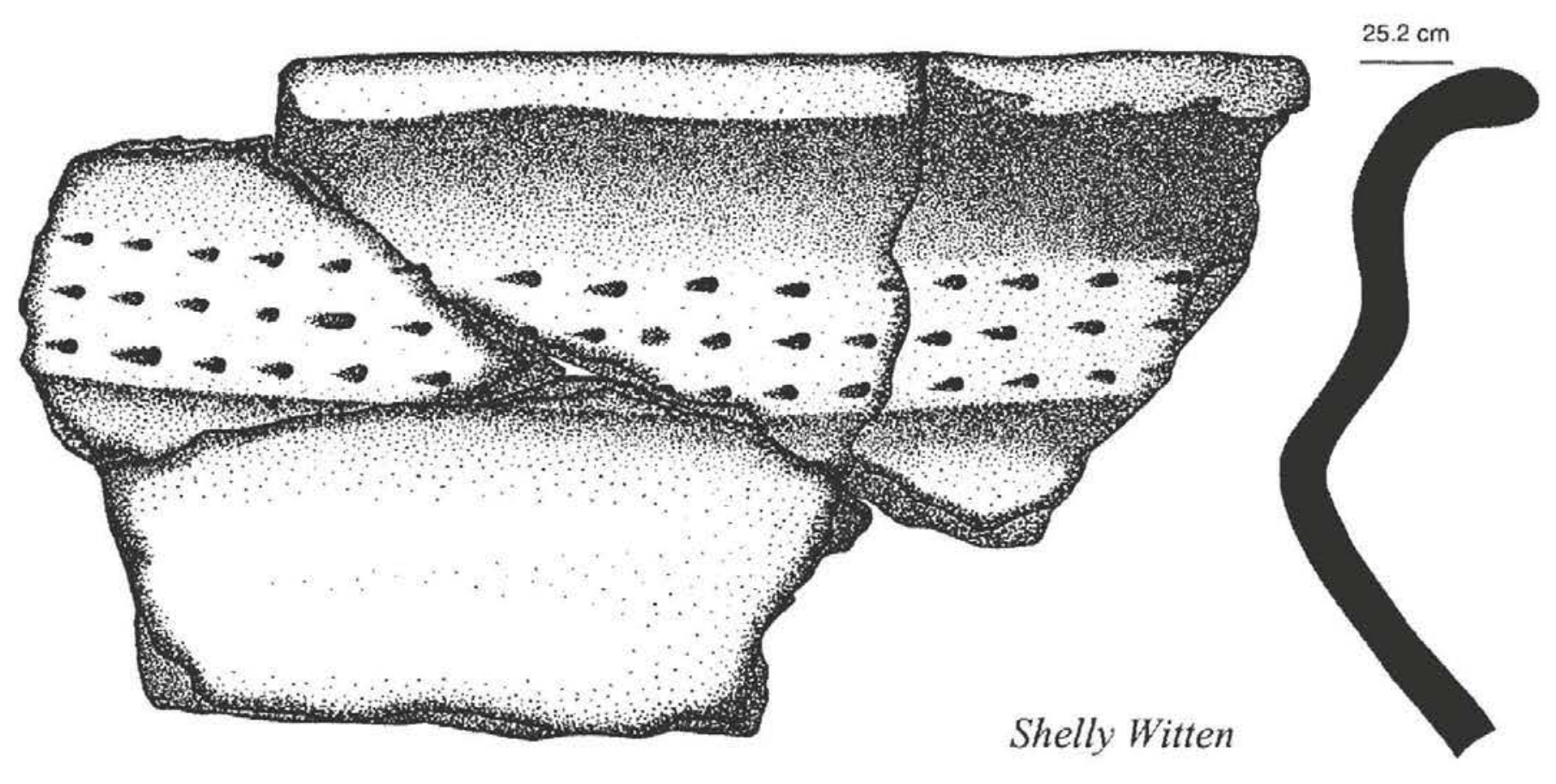




\section{Constricted Neck Punctated}

(Gregory 2005 ms.)
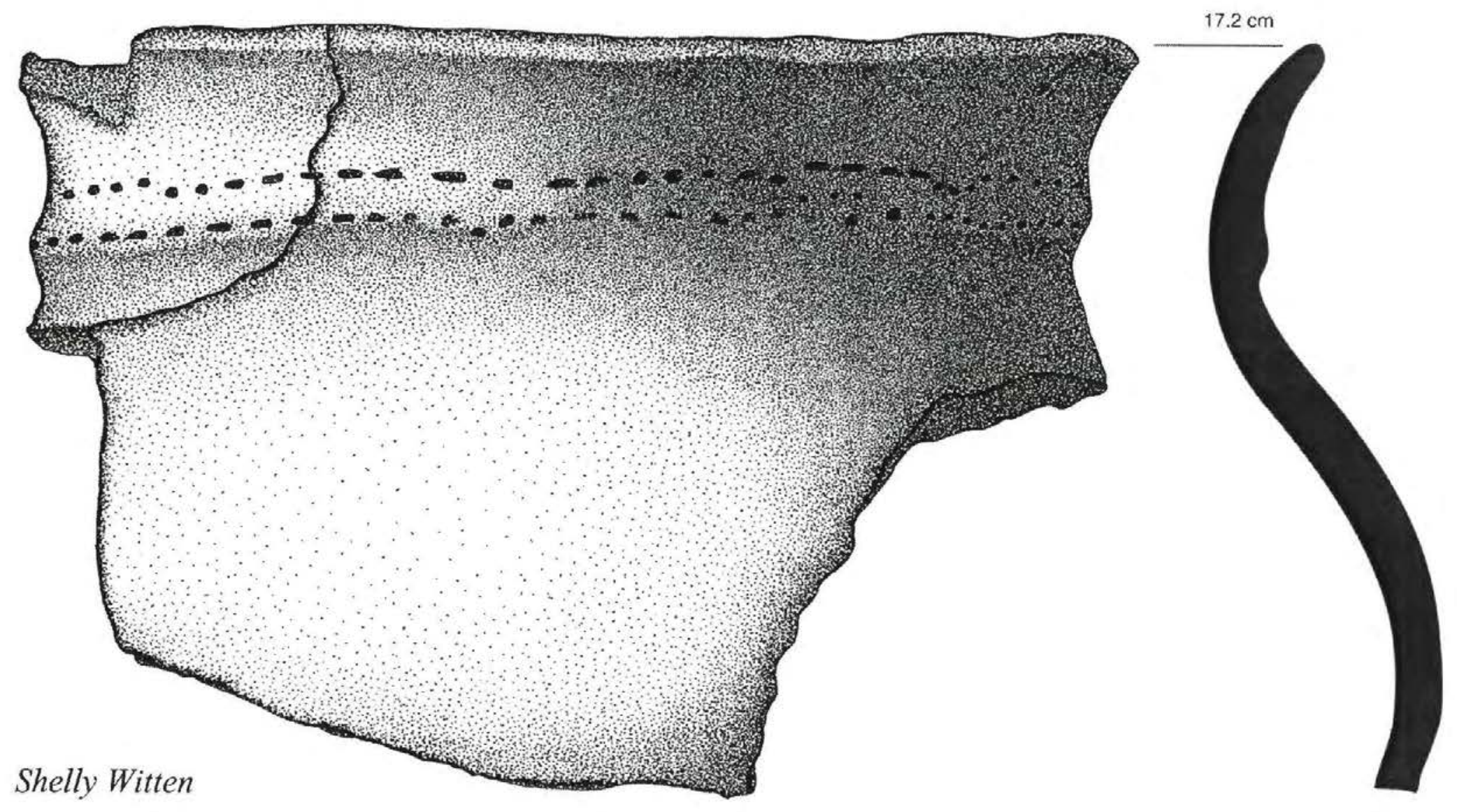


\section{Constricted Neck Punctated}

(Gregory 2005 ms.)

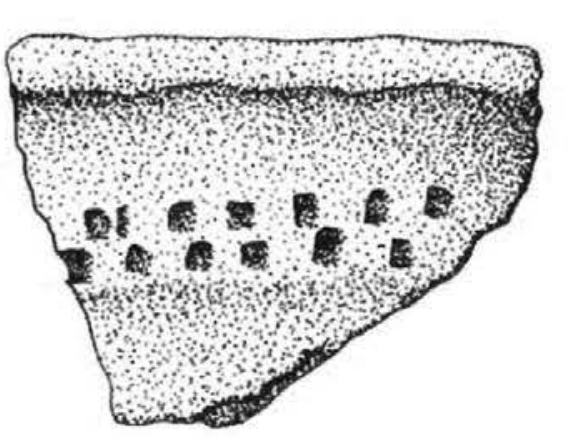

Shelly Witten


Shelly Witten

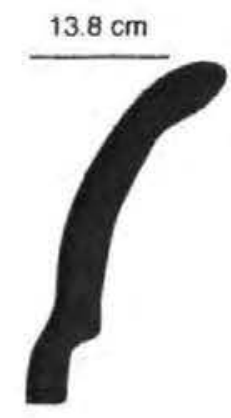

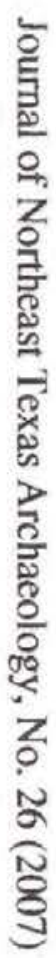



Los Adaes 16NA16 


\section{Constricted Neck Punctated}

(Gregory 2005 ms.)
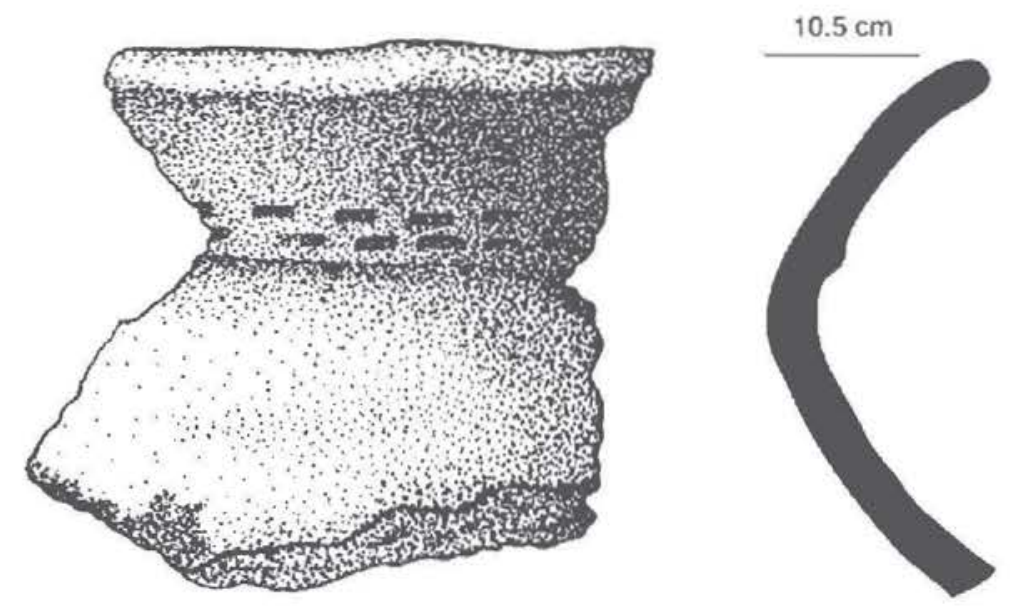

Shelly Witten
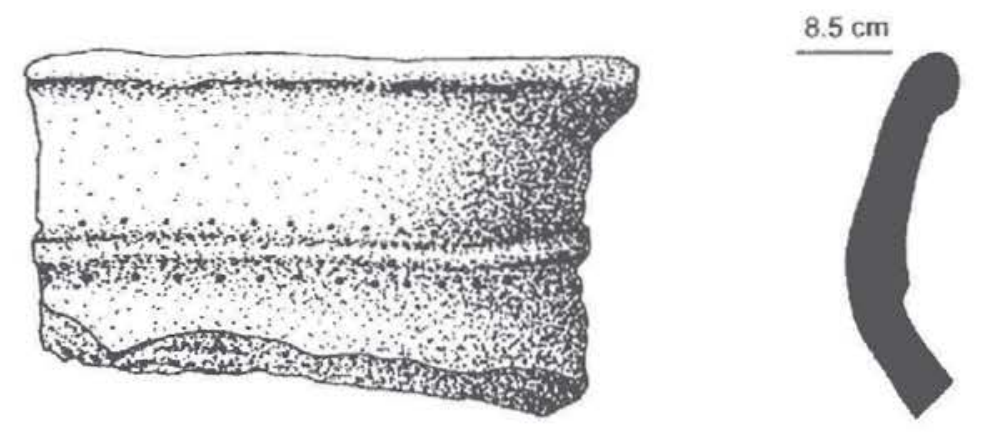

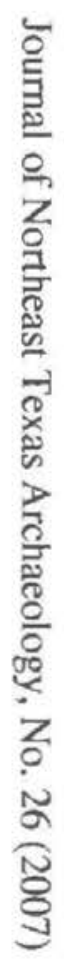

Shelly Witten






\section{Emory Punctated}

(Gregory 2005 ms.)


Shelly Witten
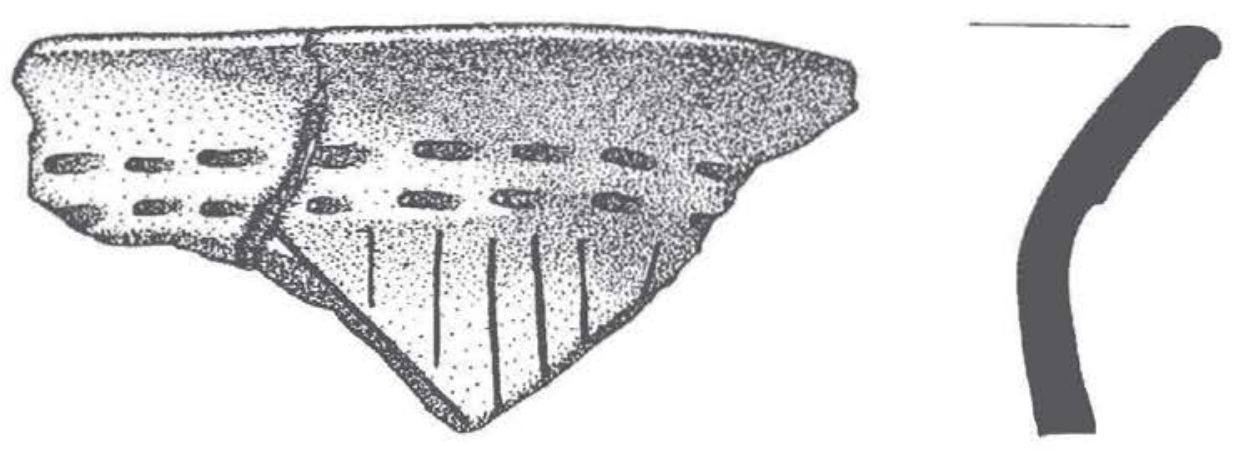

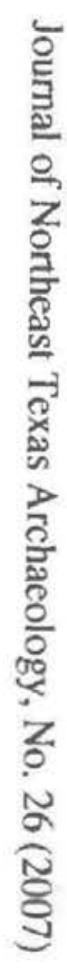

Shelly Witten 


\section{Emory Punctated}

(Gregory 2005 ms.)
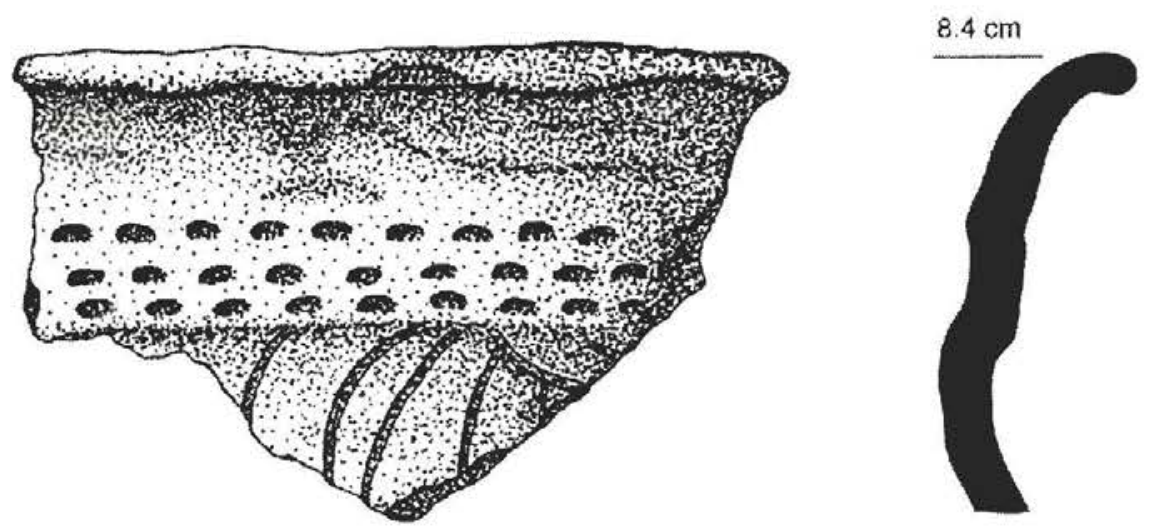

Shelly Witten
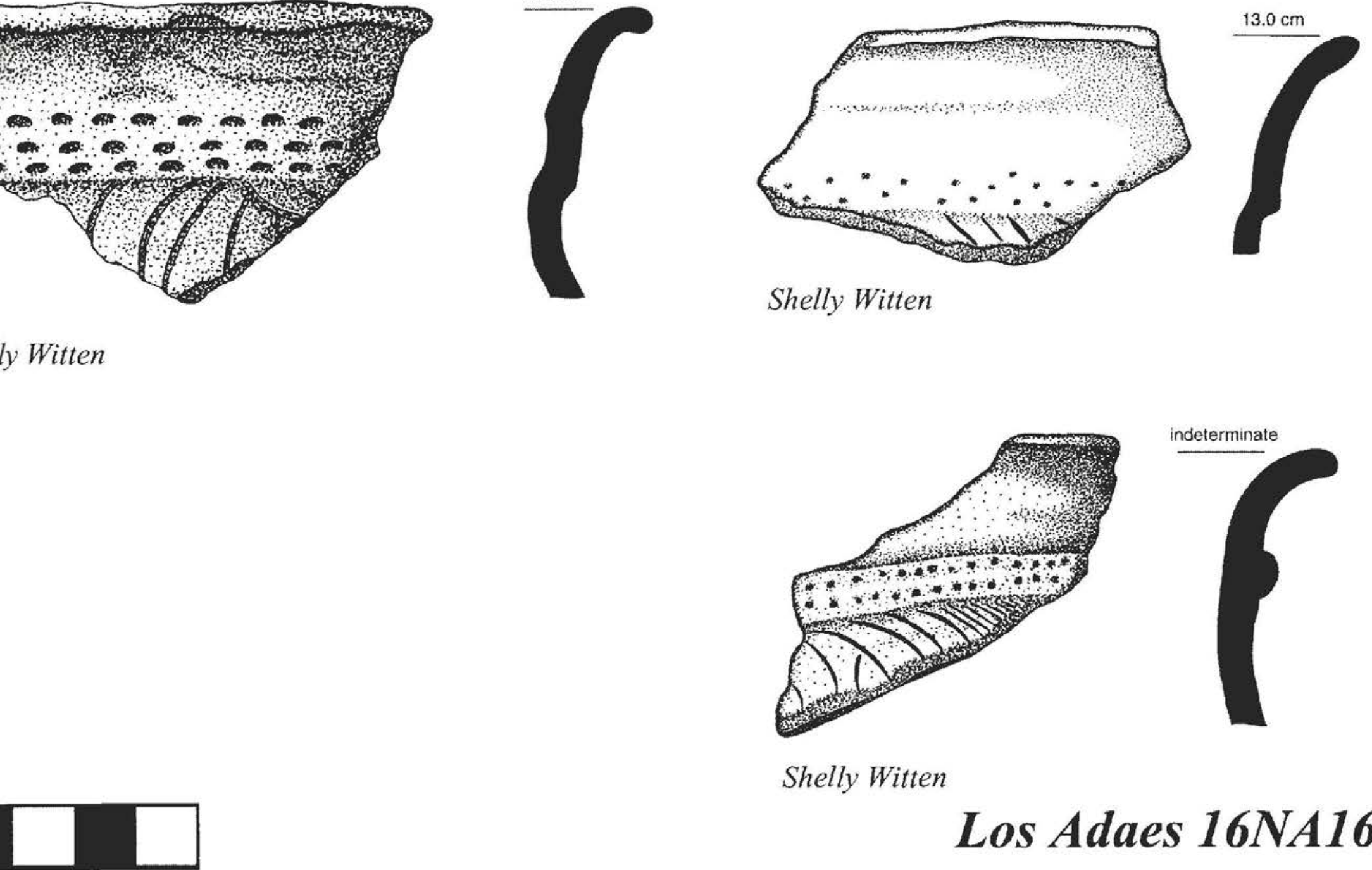

Shelly Witten

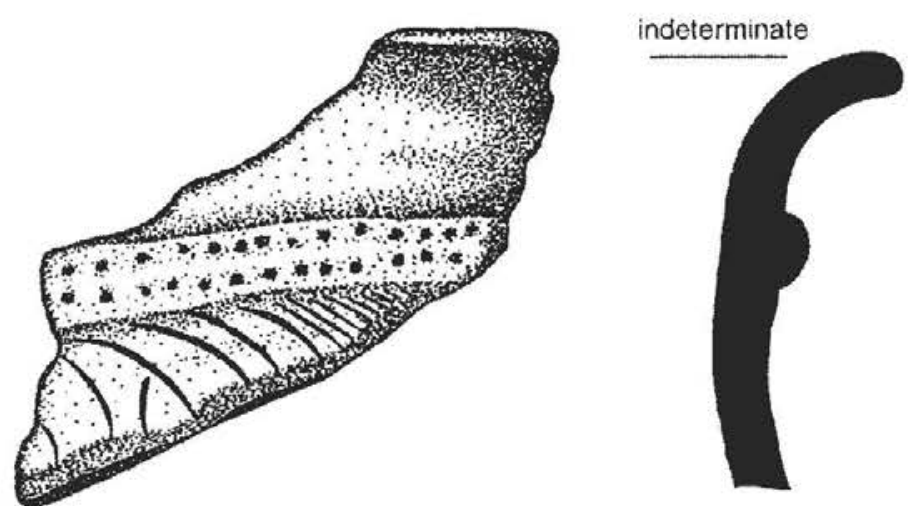

Shelly Witten 
Journal of Northeast Texas Archaeology, No. 26 (2007)

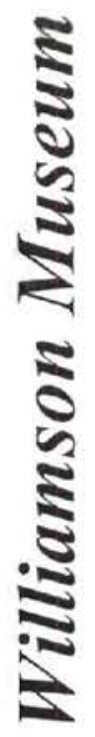
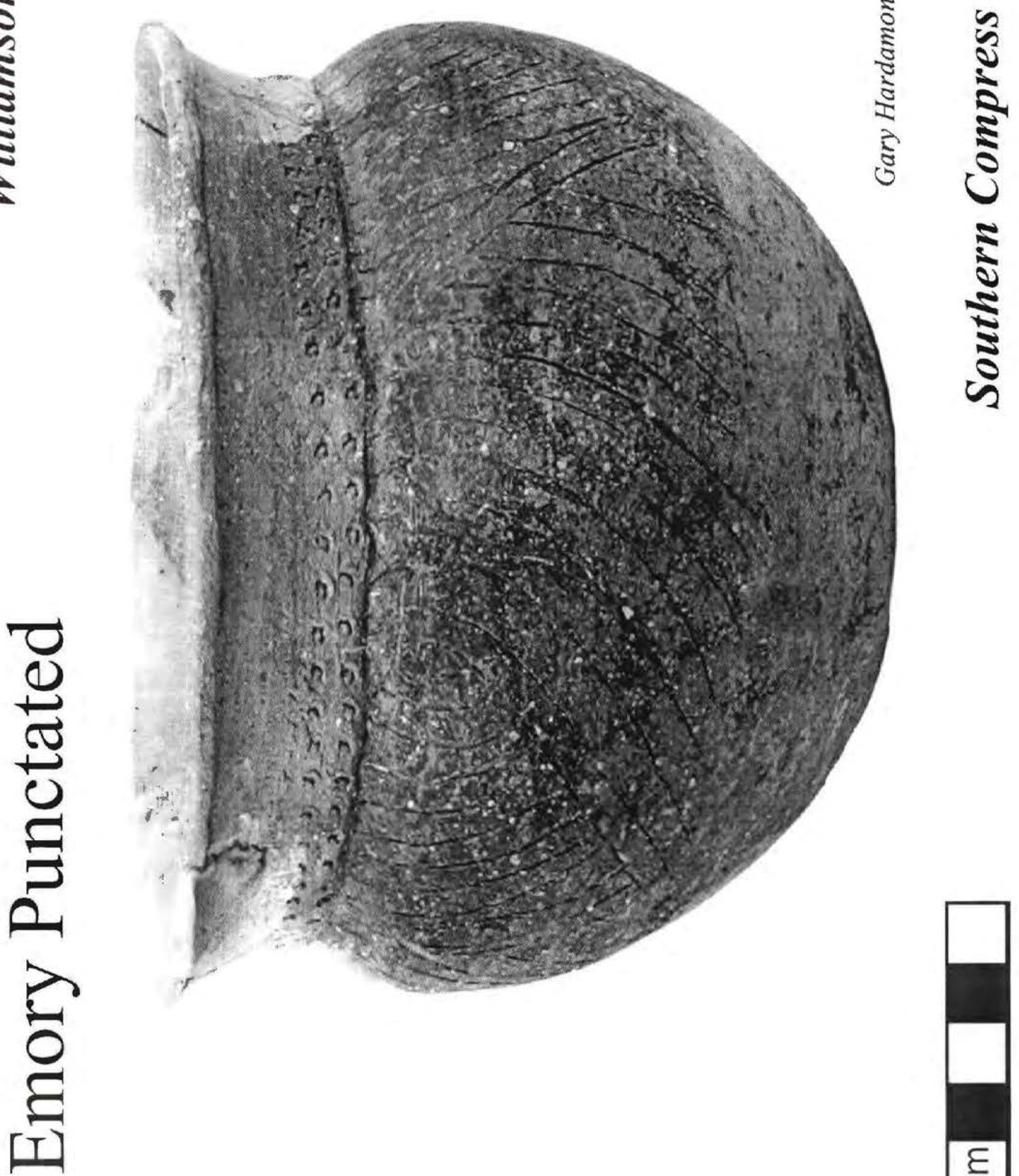


\section{Emory Punctated}

(Gregory 2005 ms.)


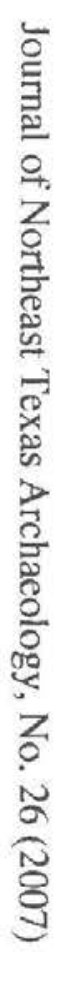




\section{Fatherland Incised}

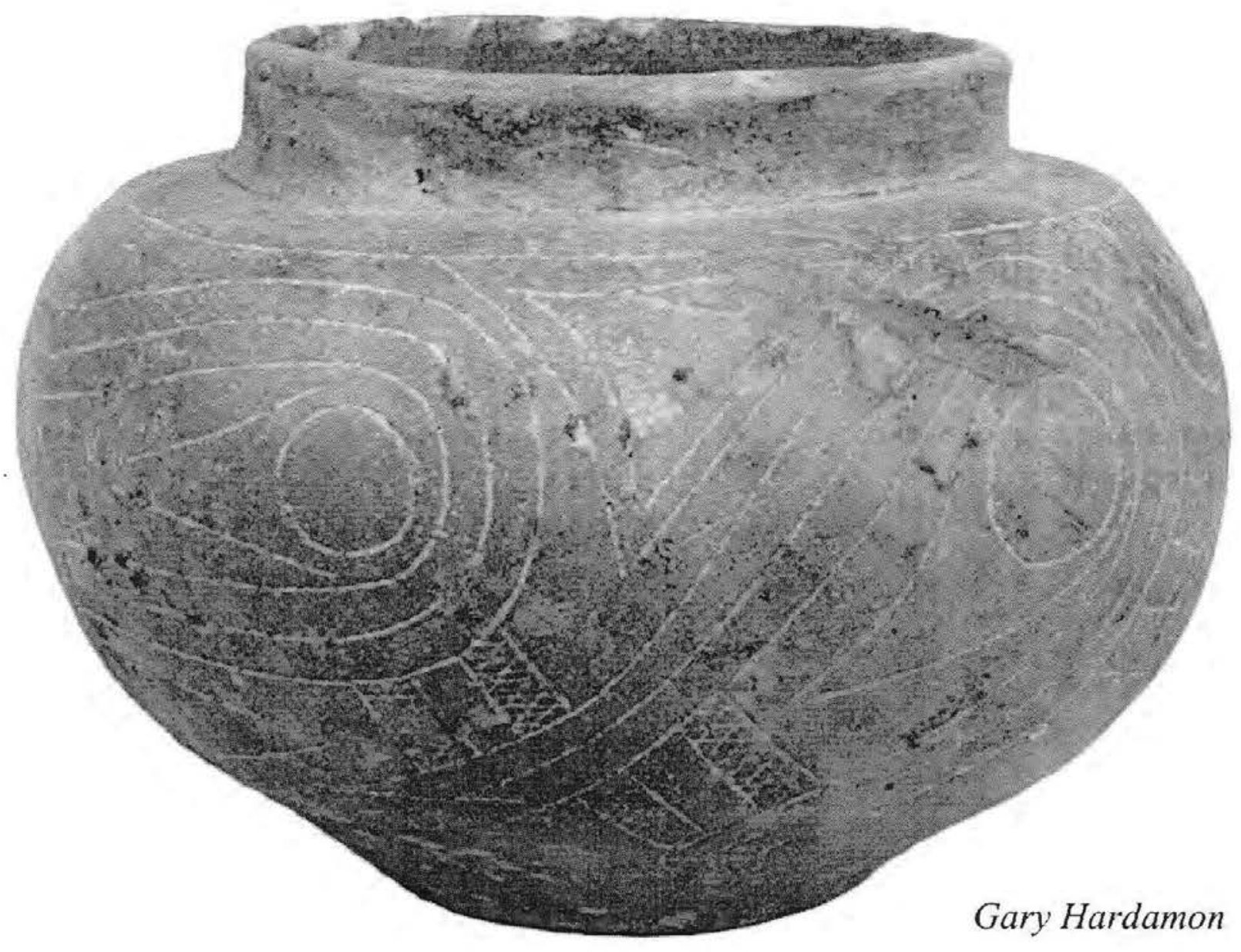



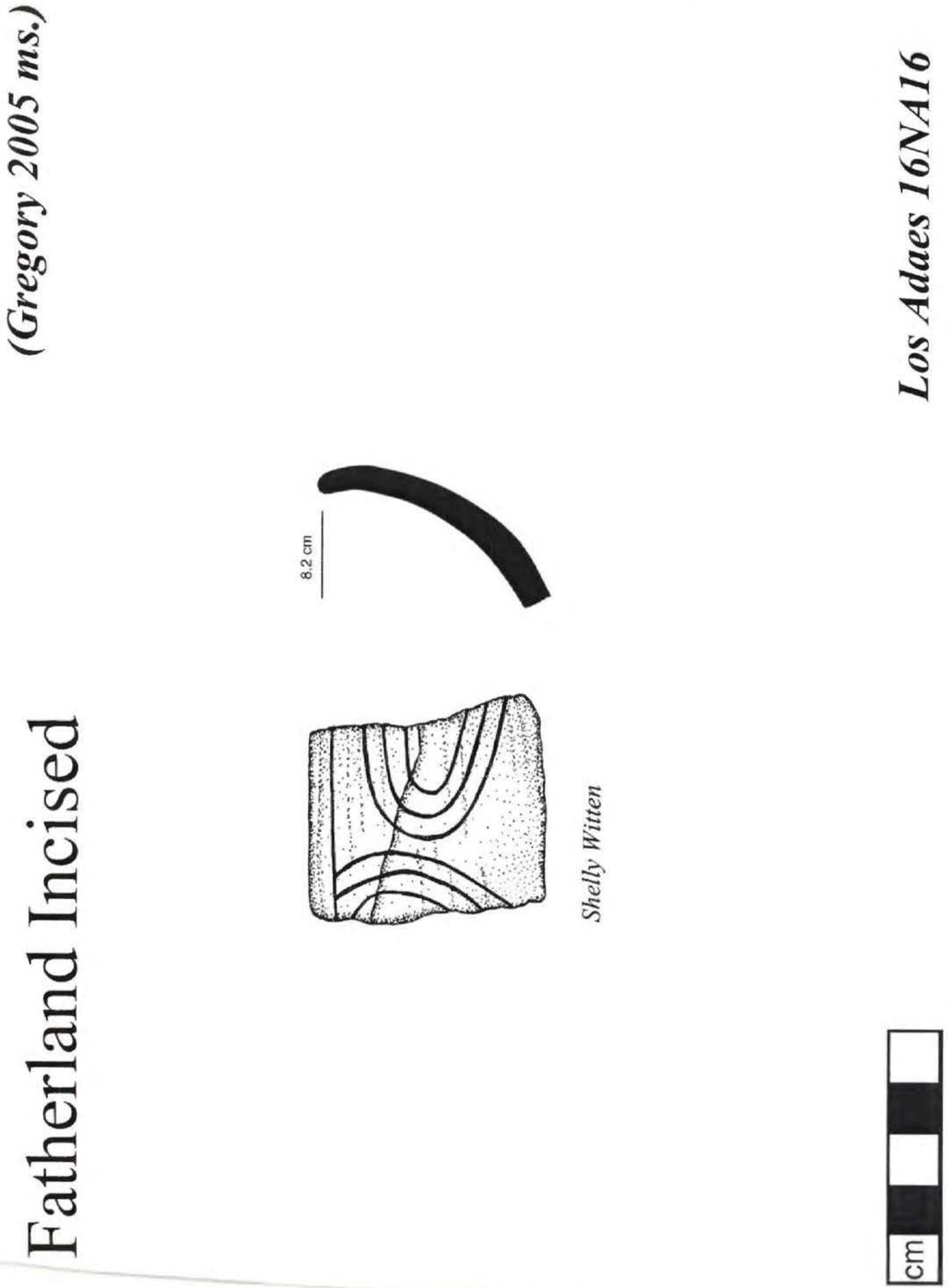

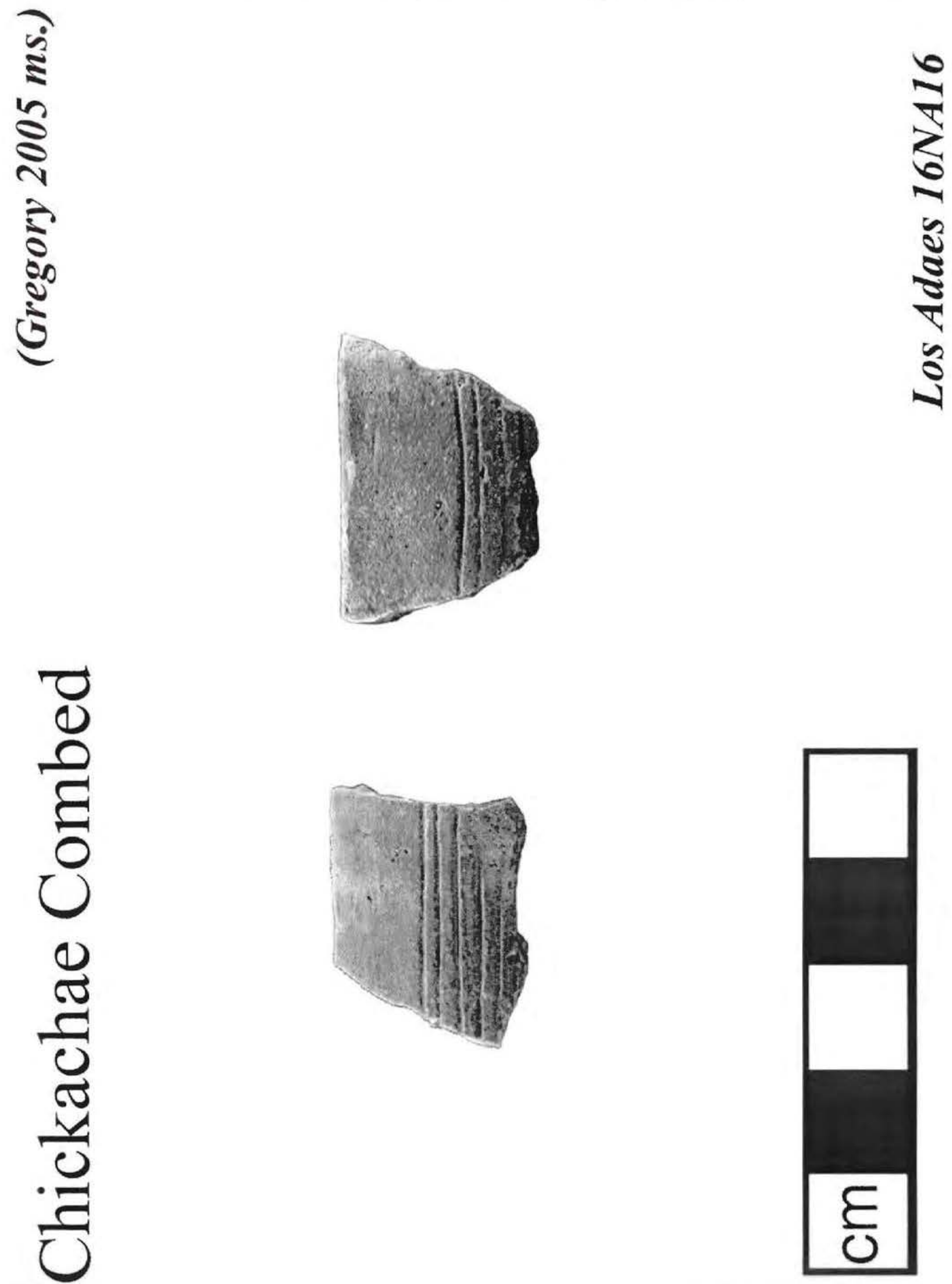


\section{"Rule of Two" Plain Wares}

(Gregory 2005 ms.)




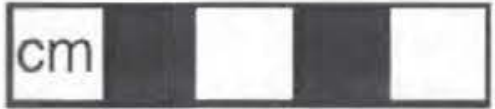

shell tempered

Los Adaes 16NA16 


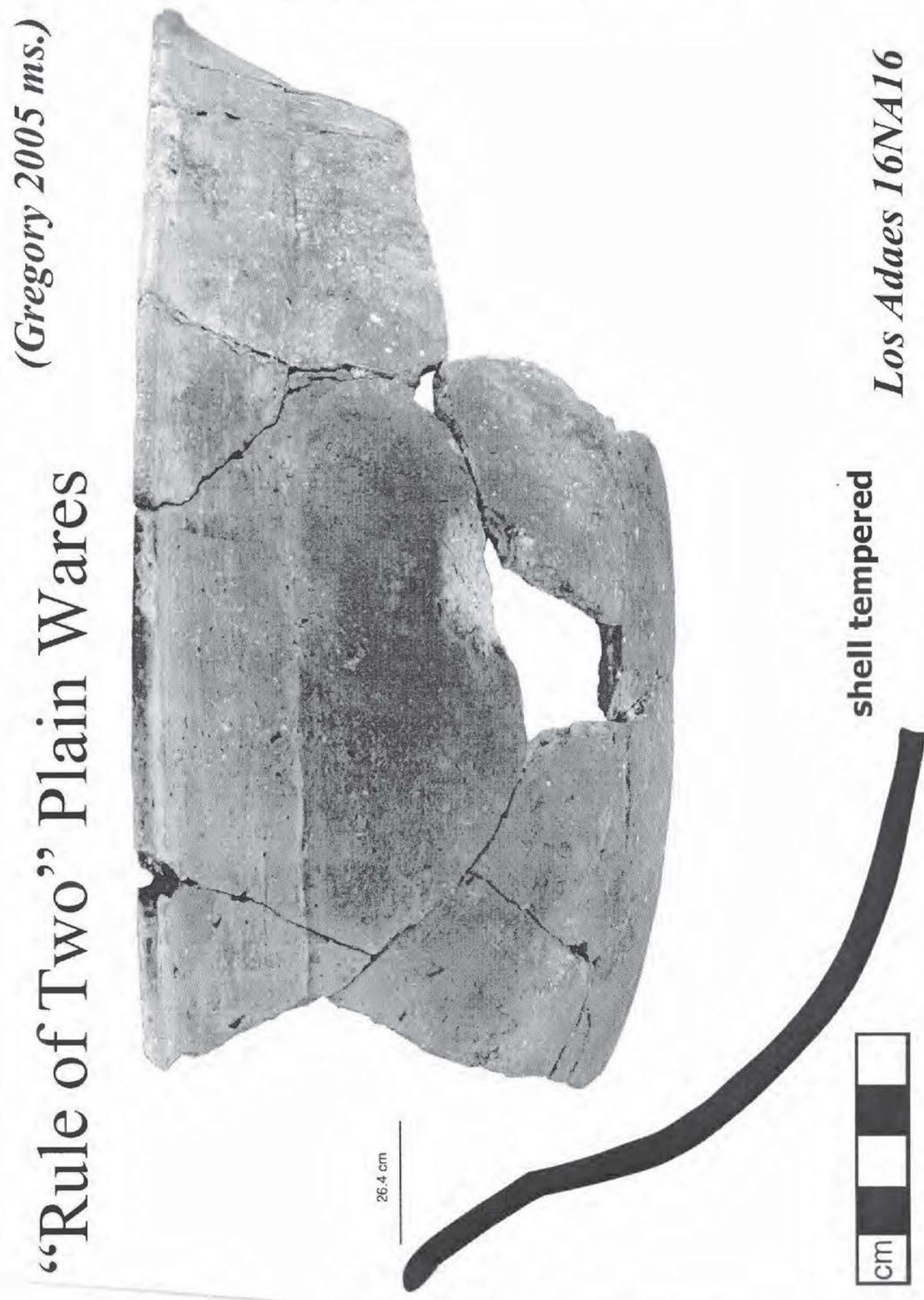




\section{"Rule of Two" Plain Wares}

(Gregory 2005 ms.)

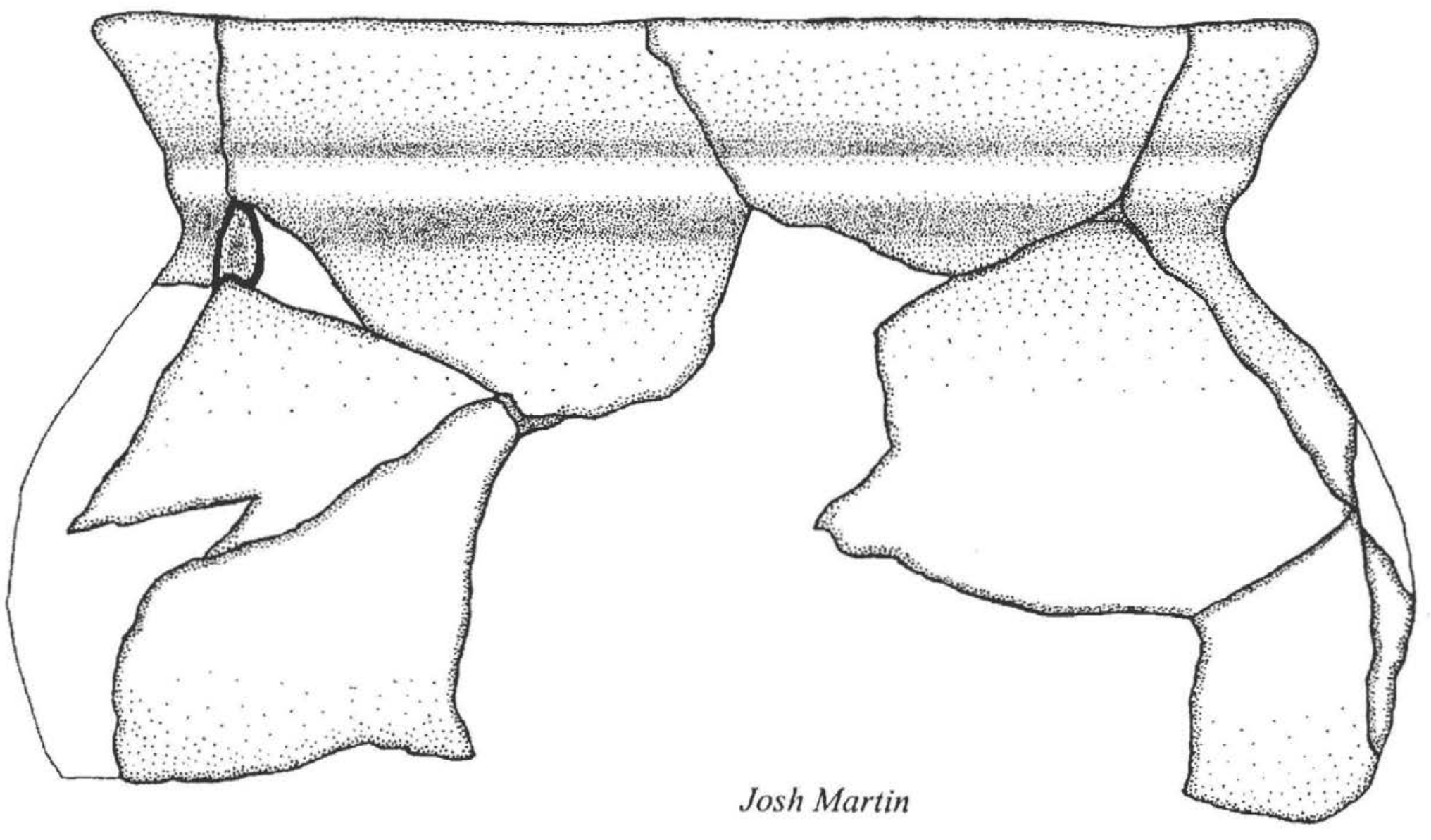



$\mathrm{cm}$ shell tempered 


\section{"Rule of Two" Plain Wares}

(Gregory 2005 ms.)

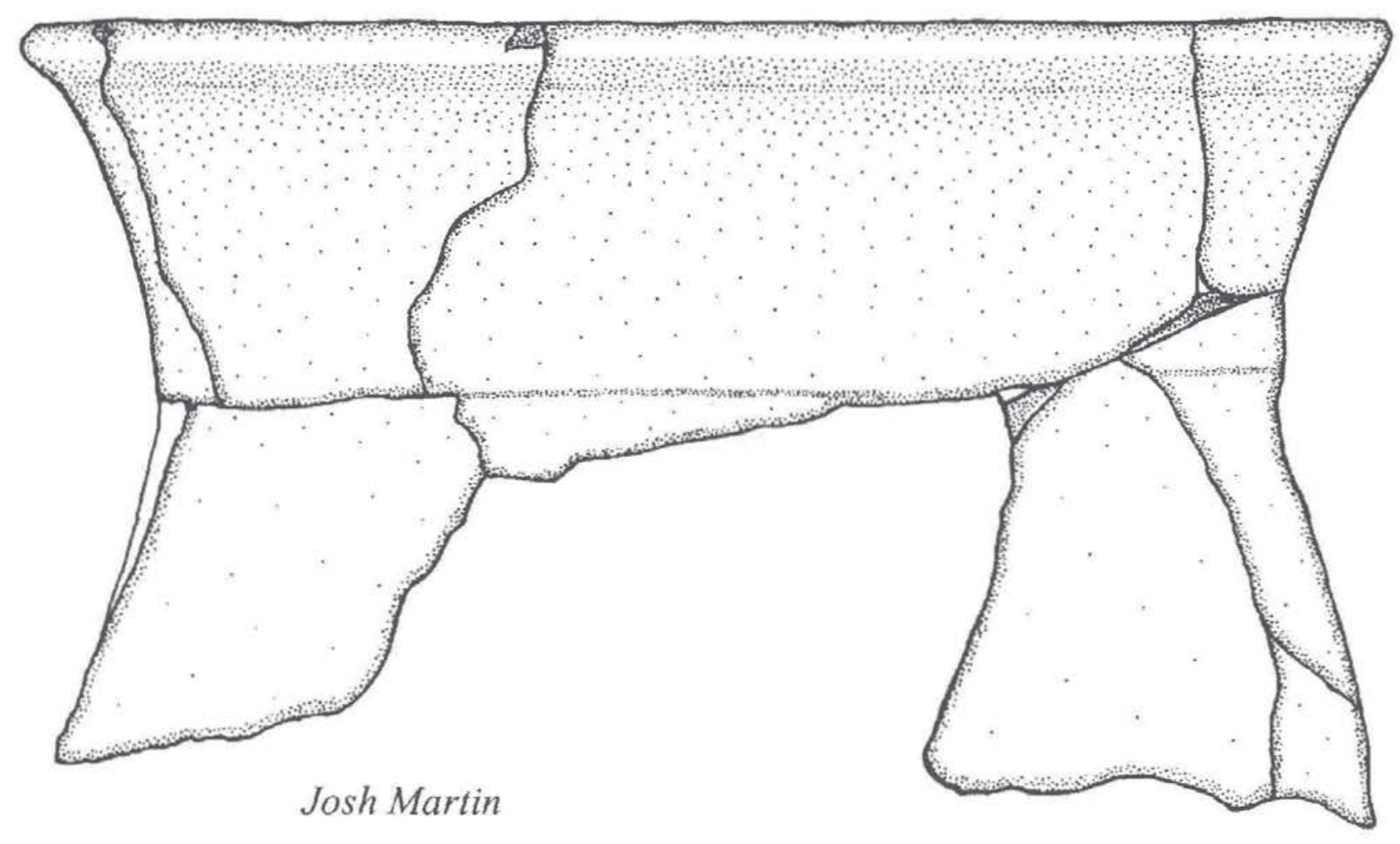




\section{"Rule of Two" Plain Wares}

(Gregory 2005 ms.)



\section{"Rule of Two" Plain Wares}

(Gregory 2005 ms.)
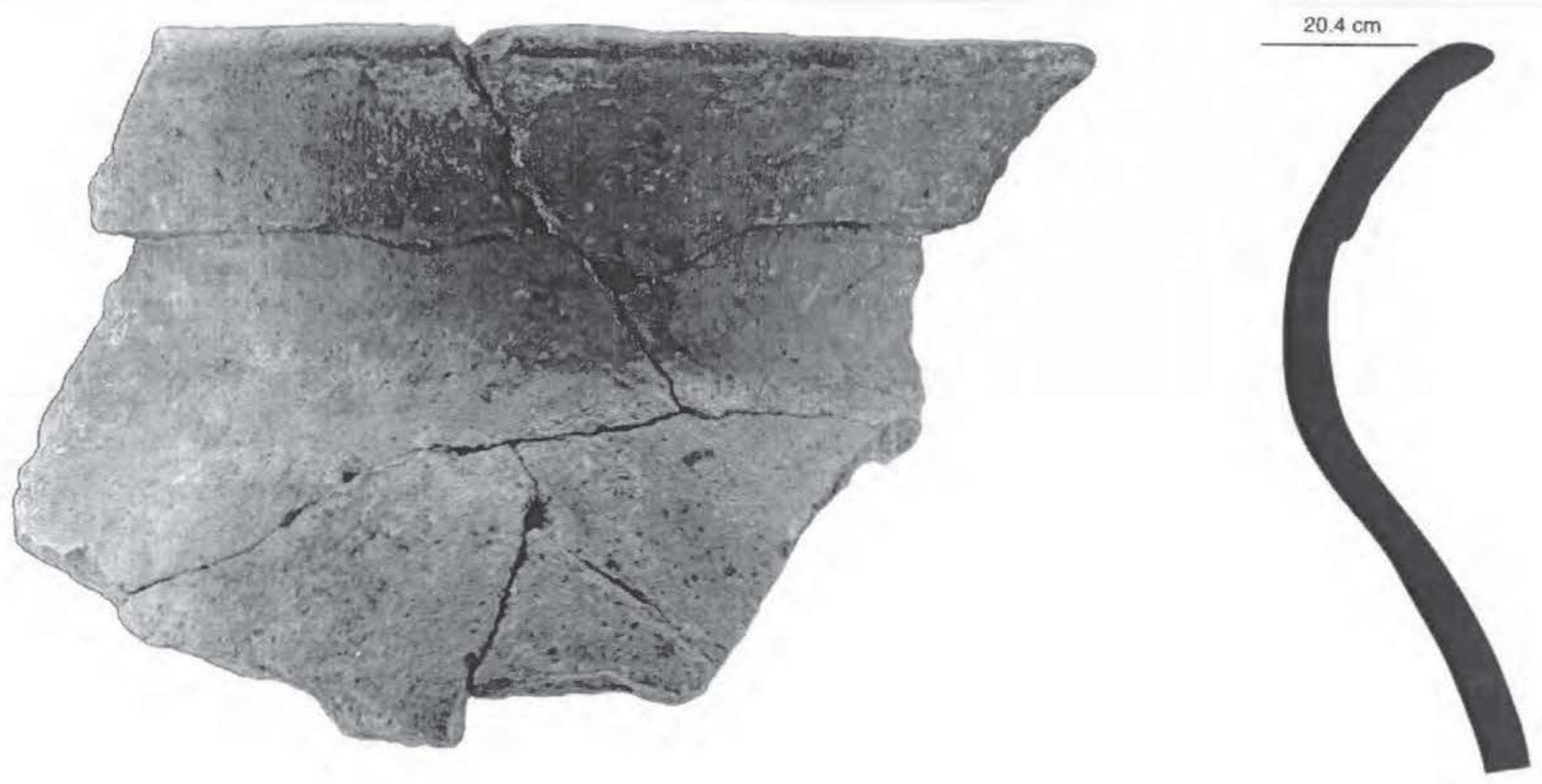

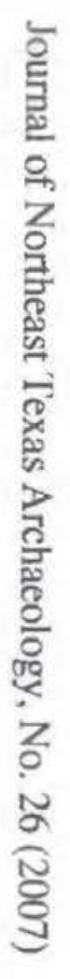

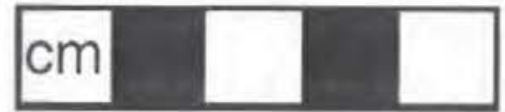

shell tempered

Los Adaes 16NA16 
0
0
0
0
0
0
0
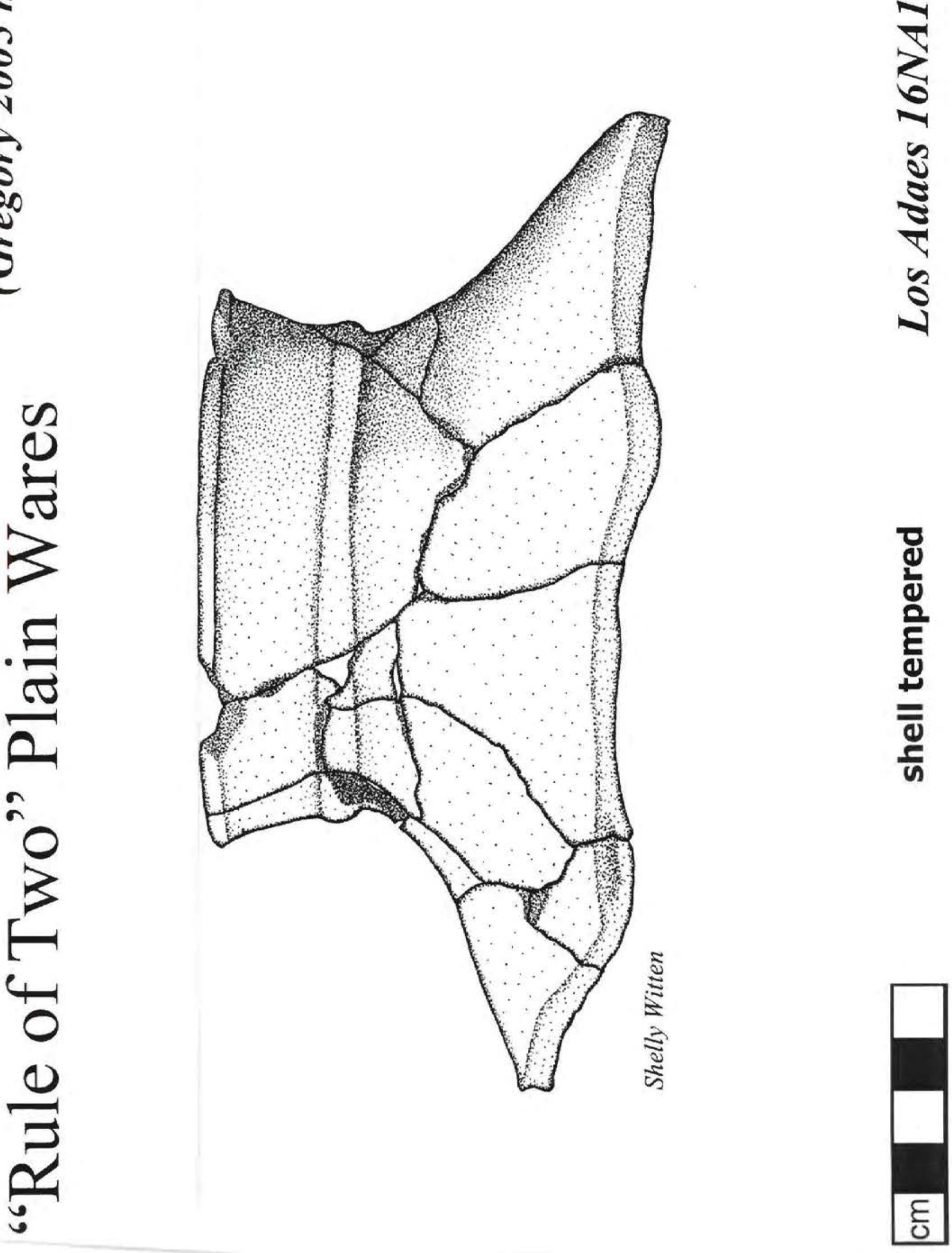

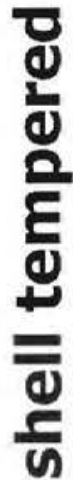


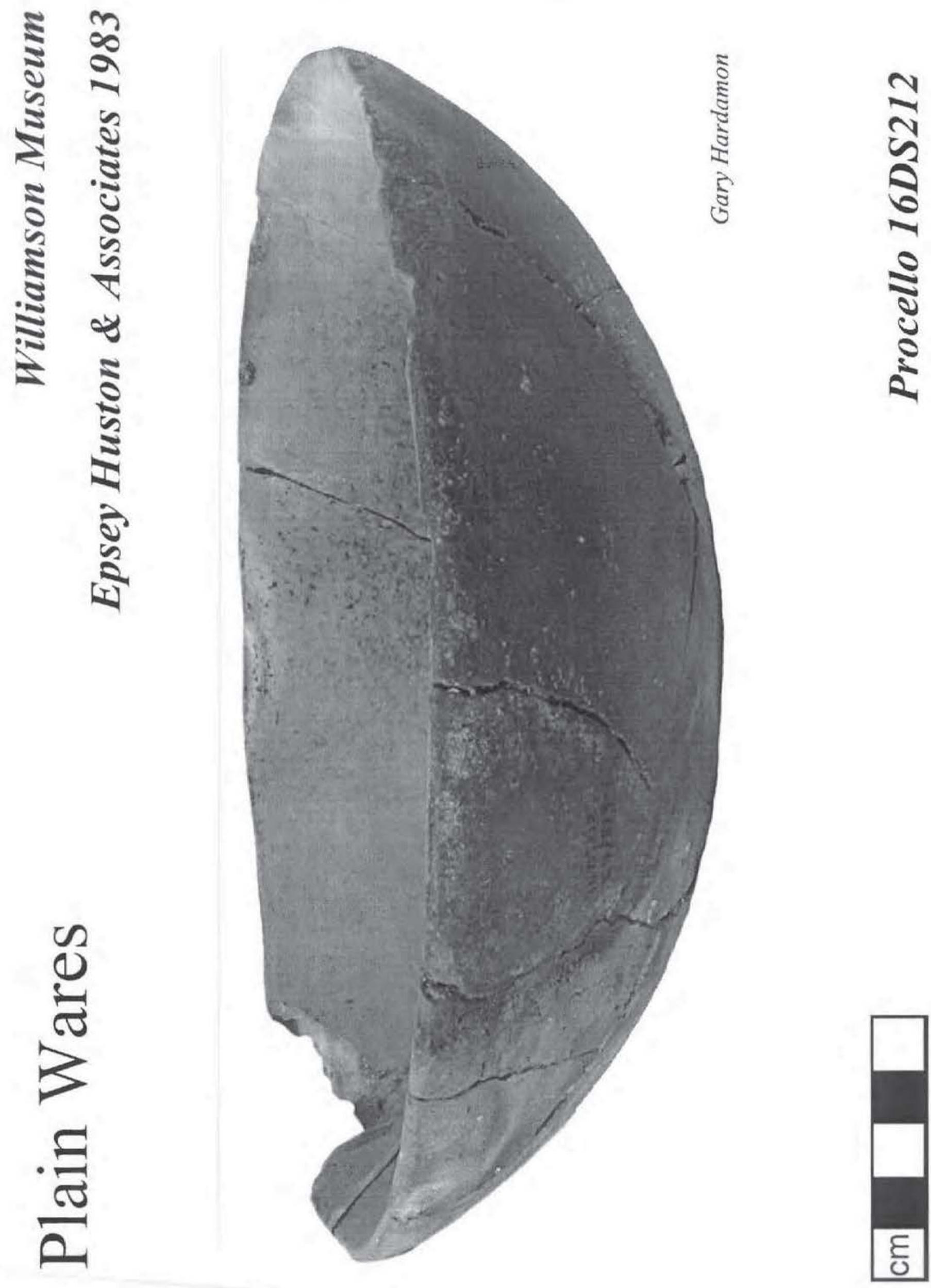


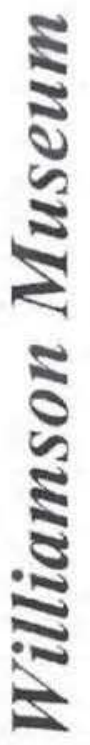

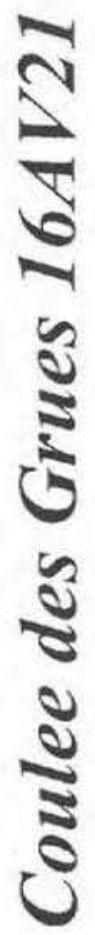

$\frac{\sigma}{2}$
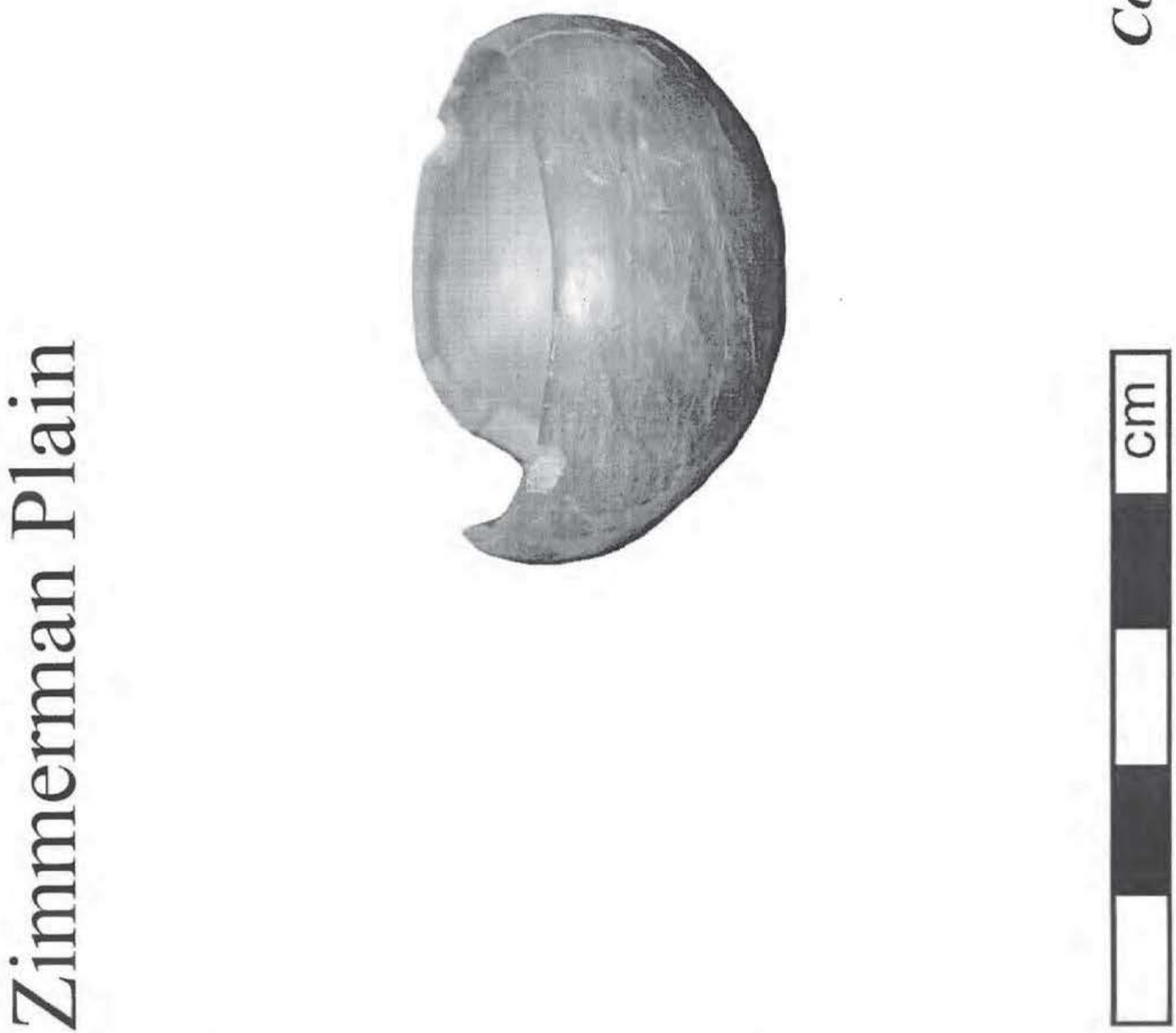
Williamson Museum

Plain Wares with European Influences

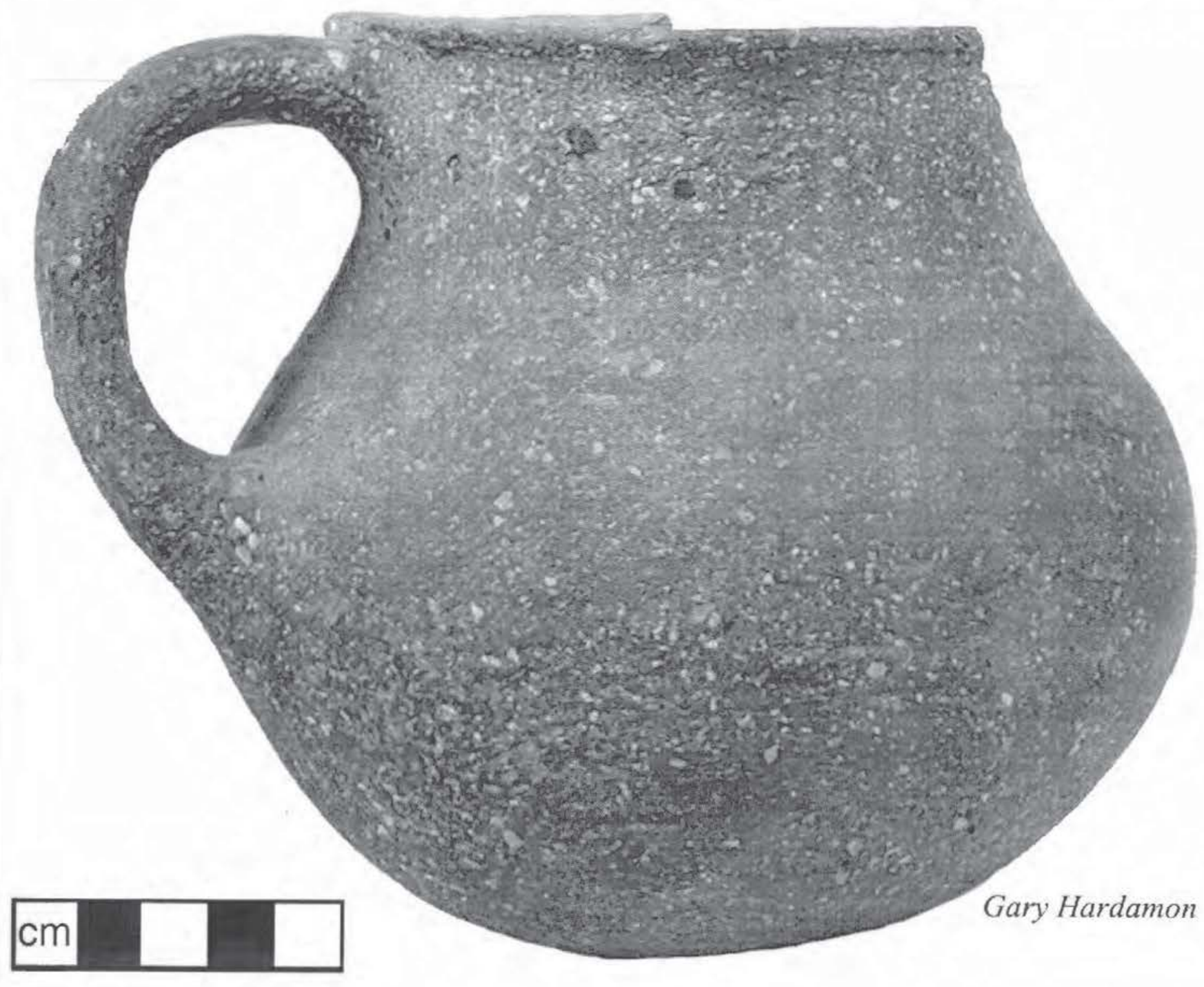

(pitcher form)

Payne

Plantation, Natchitoches

ป

Parish 
(Gregory 2005 ms.)

\section{Plain Wares with European Influences}
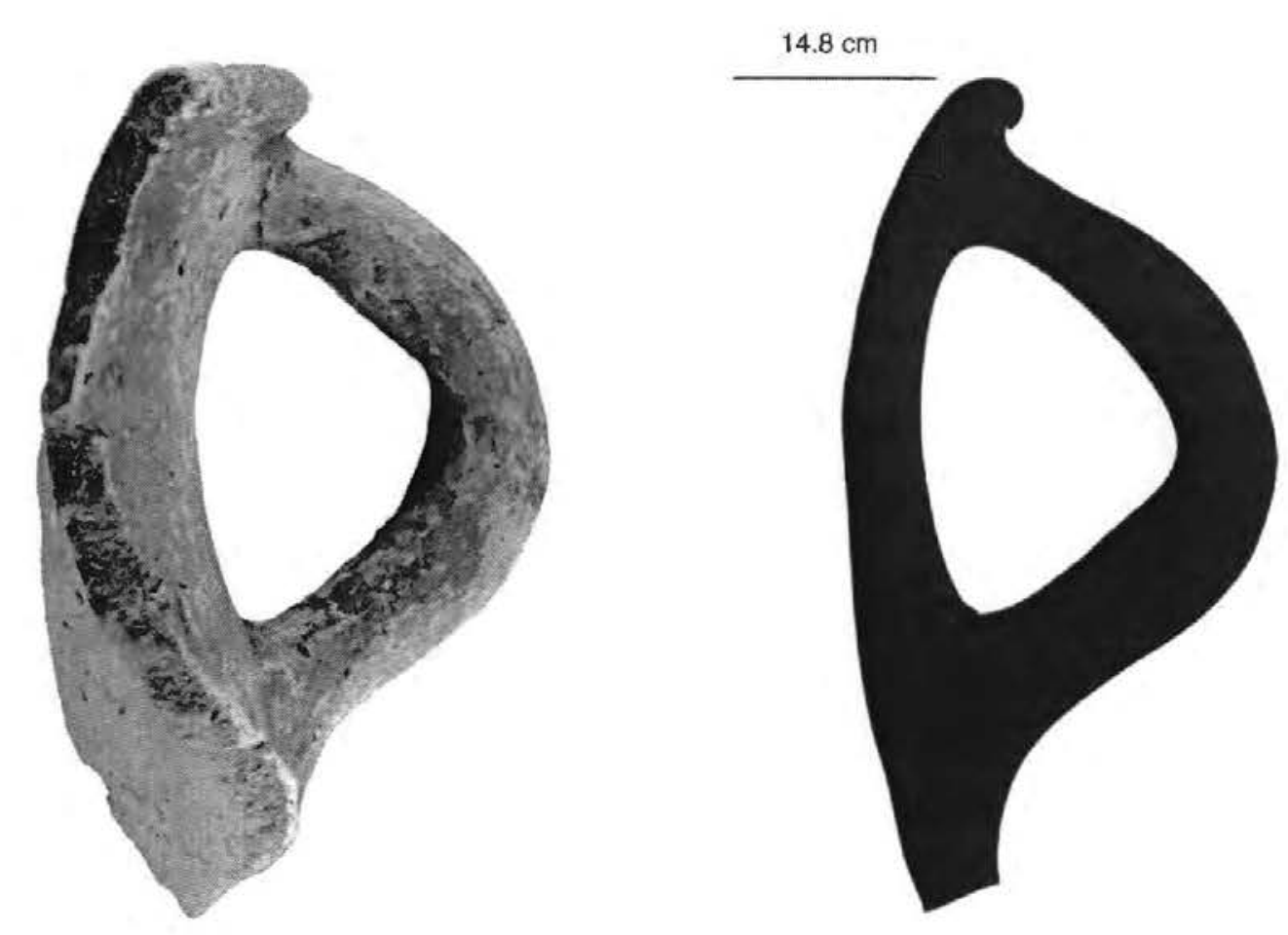




Plain Wares with European Influences (Gregory 2005 ms.) Plain Wares with European Influences
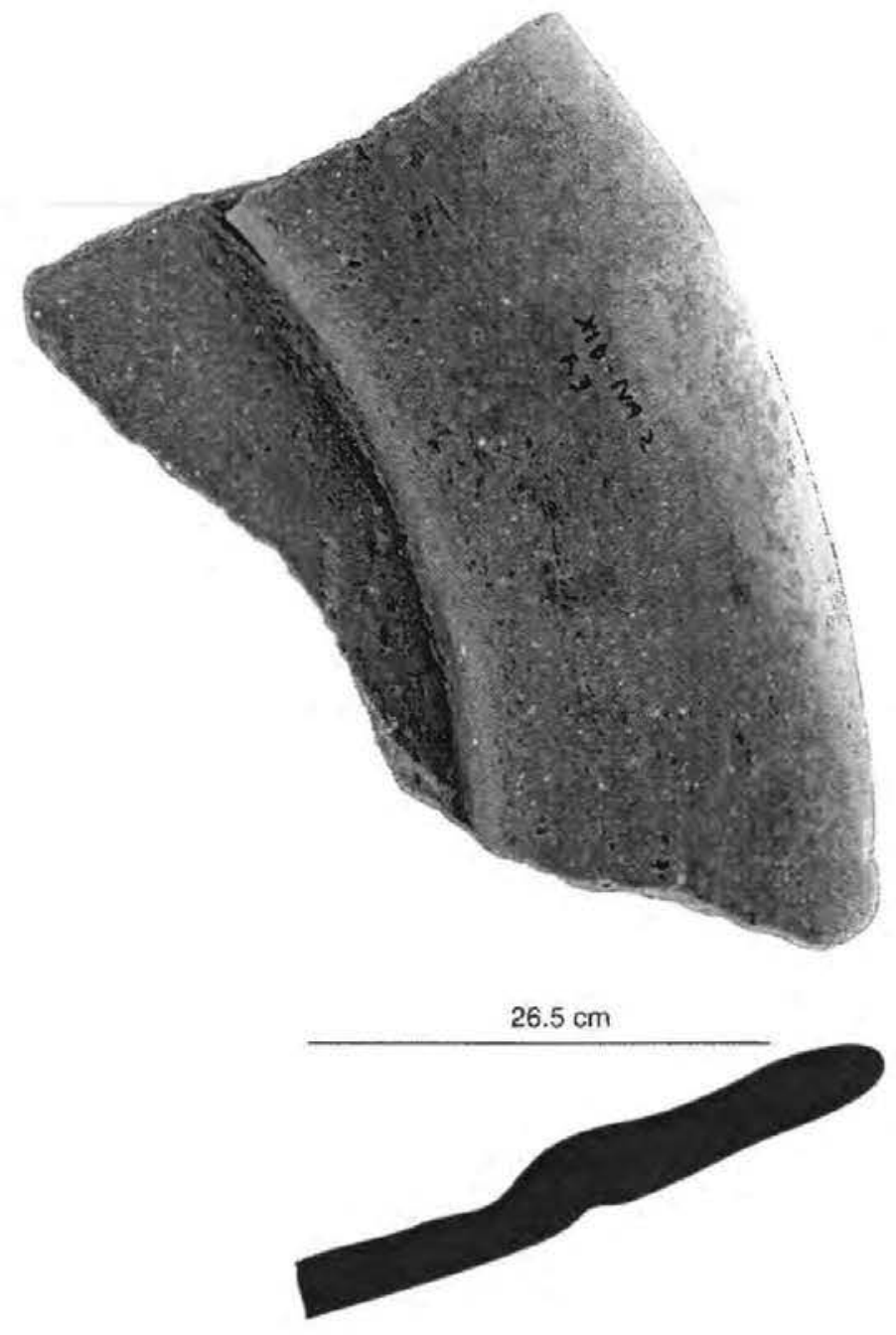



$20.0 \mathrm{~cm}$

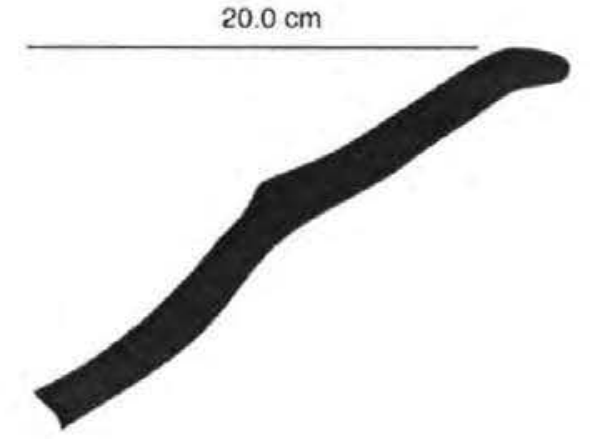


(Gregory 2005 ms.)

Plain Wares with European Influences

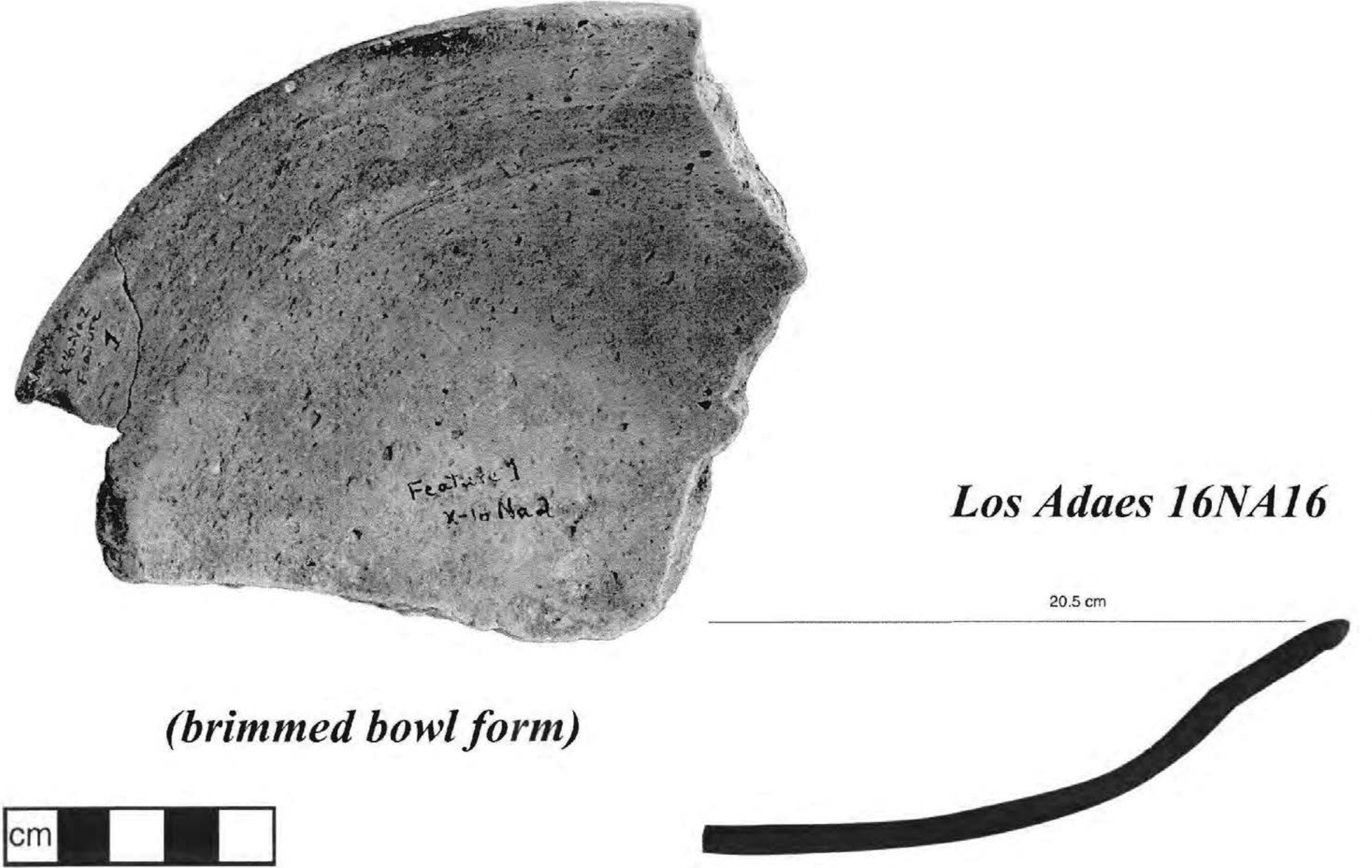




\section{Plain Wares with European Influences}

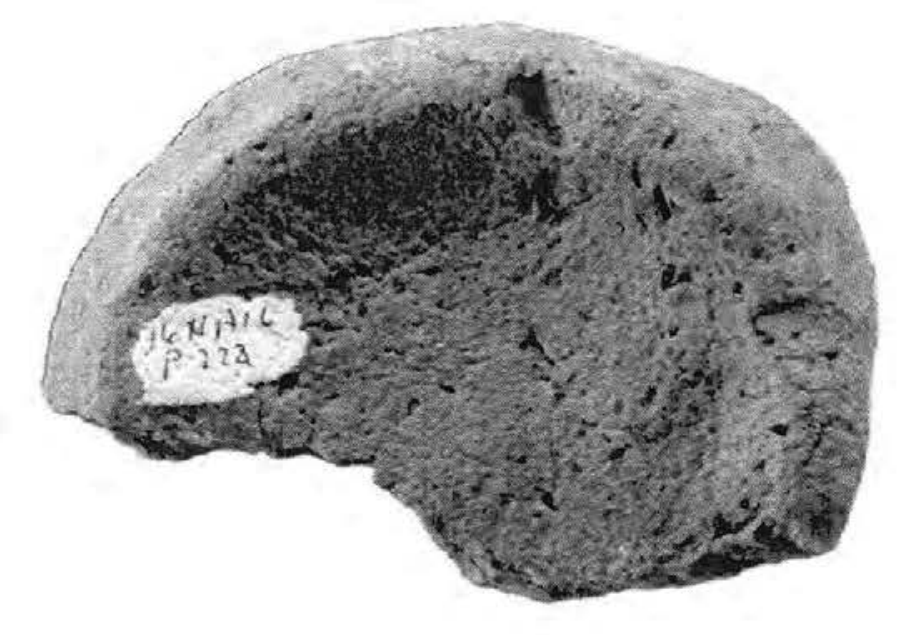

(basal sherd with foot ring)

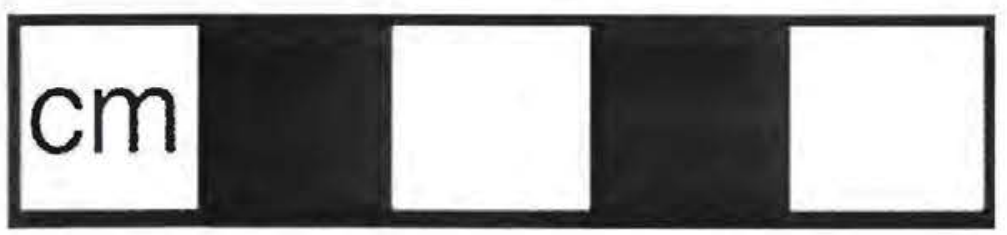




\section{Williamson Museum}

\section{Plain Wares with European Influences}



(porringer form)

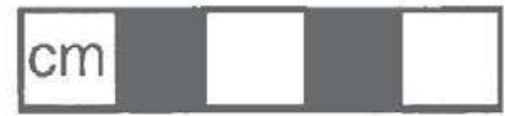

Colfax Ferry 16NA15 